\title{
GENERIC AND MAXIMAL JORDAN TYPES
}

\author{
ERIC M. FRIEDLANDER*, JULIA PEVTSOVA*, AND ANDREI SUSLIN*
}

\begin{abstract}
For a finite group scheme $G$ over a field $k$ of characteristic $p>0$, we associate new invariants to a finite dimensional $k G$-module $M$. Namely, for each generic point of the projectivized cohomological variety $\operatorname{Proj}^{\bullet}(G, k)$ we exhibit a "generic Jordan type" of $M$. In the very special case in which $G=E$ is an elementary abelian $p$-group, our construction specializes to the non-trivial observation that the Jordan type obtained by restricting $M$ via a generic cyclic shifted subgroup does not depend upon a choice of generators for $E$. Furthermore, we construct the non-maximal support variety $\Gamma(G)_{M}$, a closed subset of $\operatorname{Proj} \mathrm{H}^{\bullet}(G, k)$ which is proper even when the dimension of $M$ is not divisible by $p$.
\end{abstract}

\section{INTRODUCTION}

Elementary abelian $p$-subgroups of a finite group $G$ capture significant aspects of the cohomology and representation theory of $G$. For example, if $k$ is a field of characteristic $p>0$, then a theorem of D. Quillen [18] asserts that the Krull dimension of the cohomology algebra $\mathrm{H}^{\bullet}(G, k)$ is equal to the maximum of the ranks of elementary abelian $p$-subgroups of $G$ and a theorem of L. Chouinard [6] asserts that a $k G$-module is projective if and only if its restrictions to all elementary abelian $p$-subgroups of $G$ is projective. The cohomology algebra $\mathrm{H}^{\bullet}(E, k)$ of an elementary abelian $p$-group $E$ is easily computed, whereas $k E$ is of wild representation type provided that the rank of $E$ is at least 2 (at least 3 , for $p=2$ ). Nonetheless, Chouinard's theorem and Quillen's geometric description of $\operatorname{Spec~}^{\bullet}(G, k)$ [18] provide the basis for interesting invariants of $k G$-modules, most notably the cohomological support variety $|G|_{M}$ of a $k G$-module $M$.

The investigation of the cohomology and representation theory of finite groups is naturally extended to other algebraic structures. Friedlander and B. Parshall developed a theory of support varieties for finite dimensional $p$-restricted Lie algebras $g$ over a field $k$ of characteristic $p>0$ (e.g., [9]). For restricted Lie algebras, the role of the group algebra $k G$ of the finite group $G$ is played by the restricted enveloping algebra $u(g)$. Indeed, restricted Lie algebras lead one to more interesting geometrical structures than do finite groups, and seemingly lead to stronger results. For example, the theorem of G. Avrunin and L. Scott [1] identifying the cohomological support variety $|E|_{M}$ of a finite dimensional $k E$-module $M$ for an elementary abelian $p$-group $E$ with the rank variety of J. Carlson [3] admits a formulation in the case of a restricted Lie algebras $g$ in terms of closed subvarieties of the $p$-nilpotent cone of $g([9],[14],[20])$.

Finite groups and finite dimensional $p$-restricted Lie algebras are examples of finite group schemes. The representation theory of a finite group scheme is the study of $k G$-modules, where $k G$ is the group algebra of $G$ defined to be the $k$-linear

* partially supported by the NSF. 
dual of the representing Hopf algebra $k[G]$. A fundamental theorem of Friedlander and Suslin [13] is that the cohomology algebra $\mathrm{H}^{*}(G, k)$ is finitely generated for any such finite group scheme.

If $\mathcal{G}$ is an affine algebraic group over $k$, then the $r^{\text {th }}$ Frobenius kernel $G=$ $\mathcal{G}_{(r)} \equiv \operatorname{ker}\left\{F^{r}: \mathcal{G} \rightarrow \mathcal{G}^{(r)}\right\}$ is an infinitesimal group scheme (i.e. a connected finite group scheme). Such Frobenius kernels play an important role in the study of (rational) representations of $\mathcal{G}$ : the family $\left\{\mathcal{G}_{(r)} ; r>0\right\}$ faithfully captures the representation theory of $\mathcal{G}$. In [19], [20], Suslin, Friedlander, and C. Bendel extend to all infinitesimal group schemes $G$ earlier work on the cohomology and support varieties of finite dimensional $p$-restricted Lie algebras (whose restricted enveloping algebras are the group algebras of infinitesimal group schemes of height 1).

In [10], [12], Friedlander and Pevtsova present a uniform approach to the study of the cohomology and related representation theory of all finite group schemes. This approach involves the use of $\pi$-points of $G$, which are finite flat maps of $K$ algebras $K[t] / t^{p} \rightarrow K G$ for field extensions $K / k$; these play the role of "cyclic shifted subgroups" in the case that $G$ is an elementary abelian $p$-group and the role of 1-parameter subgroups in the case that $G$ is an infinitesimal group scheme. In [12], the space $\Pi(G)$ of equivalence classes $\left[\alpha_{K}\right]$ of $\pi$-points $\alpha_{K}: K[t] / t^{p} \rightarrow$ $K G$ of $G$ is given a scheme structure without reference to cohomology such that $\Pi(G)$ is isomorphic as a scheme to $\operatorname{Proj} \mathrm{H}^{\bullet}(G, k)$. In particular, there is a natural bijection between such equivalence classes of $\pi$-points and homogeneous prime ideals of $\mathrm{H}^{\bullet}(G, k)$. The perspective of $\pi$-points leads to the considerations of this present paper.

As was first suggested in [8], this representation-theoretic interpretation of support varieties can be refined to determine invariants of $k G$-modules which provide more detailed information than that given by support varieties. Namely, for any $\pi$ point $\alpha_{K}: K[t] / t^{p} \rightarrow K G$, one can consider the Jordan type of $\alpha_{K}(t)$ as a nilpotent operator on $M_{K}$. As one quickly discovers, the answer may vary considerably depending on the representative $\alpha_{K}$ of an equivalence class of $\pi$-points in $\Pi(G)$. Even in the case of $G=\mathbb{Z} / p \mathbb{Z} \times \mathbb{Z} / p \mathbb{Z}$ the answer depends on the choice of generators of $E$ which in turn determines the choice of representative of a $\pi$-point (Example $2.3)$.

The following theorem (Theorem 4.2) provides a new intrinsic invariant of modular representations of finite group schemes. In particular, in the case of an elementary abelian group, this invariant is independent of the choice of generators.

Theorem 0.1. Let $G$ be a finite group scheme, let $M$ be a finite dimensional $G$ module and let $\alpha_{K}$ be a $\pi$-point of $G$ which represents a generic point $\left[\alpha_{K}\right] \in \Pi(G)$. Then the Jordan type of $\alpha_{K}(t)$ viewed as a nilpotent operator on $M_{K}$ depends only upon $\left[\alpha_{K}\right]$ and not the choice of $\alpha_{K}$ representing $\left[\alpha_{K}\right]$.

For a given generic point $\left[\alpha_{K}\right] \in \Pi(G)$ and a given finite dimensional $k G$-module $M$, we call the Jordan type of $\alpha_{K}(t)$ on $M_{K}$ the generic Jordan type of $M\left(\right.$ at $\left.\left[\alpha_{K}\right]\right)$. As we verify in Proposition 4.7, sending a module $M$ to its generic Jordan type $\left[\alpha_{K}\right]^{*}\left(M_{K}\right)$ for generic $\left[\alpha_{K}\right] \in \Pi(G)$ determines a well defined tensor triangulated functor on stable module categories $\left[\alpha_{K}\right]^{*}: \operatorname{stmod}(k G) \rightarrow \operatorname{stmod}\left(K[t] / t^{p}\right)$. In other words, generic Jordan type is well-behaved with respect to short exact sequences, direct sums, tensor products, and Heller shifts.

We establish Theorem 0.1 by successively considering more general finite group schemes, beginning with elementary abelian $p$-groups and then abelian finite group 
schemes in $\S 2$ and then infinitesimal group schemes in $\S 3$ before treating the general case in $\S 4$. The following theorem (Theorem 1.12) concerning commuting nilpotent matrices provides the key to our proof of Theorem 0.1 .

Theorem 0.2. Let $k$ be an infinite field and let $\alpha, \alpha_{1}, \ldots, \alpha_{n}$ be a set of commuting nilpotent $n \times n$ matrices such that the Jordan type of $\alpha$ is greater or equal to the Jordan type of any $\alpha+c_{1} \alpha_{1}+\cdots+c_{n} \alpha_{n}$ for all $c_{i} \in k$. Then the Jordan type of $\alpha$ is greater or equal to the Jordan type of any polynomial in $\alpha, \alpha_{1}, \ldots, \alpha_{n}$ without constant term.

For a given $k G$-module $M$, we define a notion of a maximal Jordan type (Definition 2.4). Some but not necessarily all of the generic Jordan types are maximal, but maximal Jordan types are also realized at non-generic points. The locus of non-maximal Jordan types for a given finite dimensional $k G$-module $M$ provides us with a geometric invariant $\Gamma(G)_{M}$ which agrees with the support variety of $M$ if and only if the maximal type of $M$ is projective. (Theorems 4.10, 5.2).

Theorem 0.3. Let $G$ be a finite group scheme over $k$ and $M$ a finite dimensional $k G$-module. Then whether or not the Jordan type of $\alpha_{K}^{*}\left(M_{K}\right)$ is maximal for $M$ depends only upon the equivalence class of $\alpha_{K},\left[\alpha_{K}\right] \in \Pi(G)$. The set of those equivalence classes of $\pi$-points $\alpha_{K}: K[t] / t^{p} \rightarrow K G$ such that the Jordan type of $\alpha_{K}^{*}(M)$ is not maximal for $M$ is a closed subvariety

$$
\Gamma(G)_{M} \subset \Pi(G)
$$

of $\Pi(G) \simeq \operatorname{Proj}^{\bullet}(G, k)$.

The prototype of our non-maximal support variety $\Gamma(G)_{M}$ was introduced by W. Wheeler ([22]) for elementary abelian $p$-groups. Our construction significantly strengthens Wheeler's result even in the case of an elementary abelian $p$-group $E$, since we do not rely upon a fixed set of generators for $E$.

The reader will find various examples throughout the paper intended to illustrate some of these new invariants and their behavior. More sophisticated examples which reflect deeper properties of the representation theory of $G$ are considered in [4].

In $\S 1, k$ will denote an infinite field and in subsequent sections $k$ will denote an arbitrary field of characteristic $p>0$. If $M$ is a $k$-vector space and $K / k$ is a field extension, we use the notation $M_{K}$ to denote $M \otimes_{k} K$. If $M$ is a finite dimensional $k$ vector space equipped with the structure of a $k[t] / t^{p}$-module, then the data of the block sizes of $M$ as a $k[t] / t^{p}$-module (or, equivalently, of the Jordan form of $t$ viewed as an endomorphism of $M$ ) will be called the Jordan type of $M$. In particular, the Jordan type of the $k[t] / t^{p}$-module $M$ is the same as that of the $K[t] / t^{p}$-module $M_{K}$ for any field extension $K / k$. We employ the notation $\mathrm{H}^{\bullet}(G, k)$ for the commutative $k$-algebra given as the full cohomology algebra $\mathrm{H}^{*}(G, k)$ of $G$ if $\operatorname{char}(k)=2$ and for the subalgebra of $\mathrm{H}^{*}(G, k)$ generated by classes homogeneous of even degree if $p \neq 2$. For a map $f: H \rightarrow G$ of finite group schemes, we denote by the same letter $f$ the induced map on group algebras, $f: k H \longrightarrow k G$, and by $f^{*}: G$-mod $\longrightarrow$ $H$-mod the pull-back via $f$ on the categories of modules.

We are pleased to acknowledge useful conversations with Steve Smith. Friedlander and Suslin gratefully acknowledge the support of the Institute for Advanced Study, and Friedlander and Pevtsova gratefully acknowledge the hospitality of the Max-Planck Institut in Bonn. 


\section{Maximality for COMmuting nilpotent matrices}

In this section, we consider a finite set of pair-wise commuting nilpotent matrices $\alpha_{1}, \ldots, \alpha_{n}$ with coefficients in an infinite field $k$. Theorem 1.12 establishes that maximality of the Jordan type among all polynomials in the $\alpha_{i}{ }^{\prime} \mathrm{s}$ is realized by some linear combination of the $\alpha_{i}{ }^{\prime}$ s. This theorem is the essential step in our investigation in later sections of maximality of Jordan types associated to finite dimensional representations of finite group schemes.

Given two partitions $\underline{n}=n_{1} \geq n_{2} \geq \ldots \geq n_{N}$, and $\underline{m}=m_{1} \geq m_{2} \geq \ldots \geq m_{N}$ of $N$ we say that $\underline{n}$ dominates $\underline{m}$, or $\underline{n} \geq \underline{m}$, if

$$
\sum_{1 \leq j \leq k} n_{j} \geq \sum_{1 \leq j \leq k} m_{j} \quad \text { for } 1 \leq k \leq N
$$

(see $[7,6.2 .1])$.

Definition 1.1. Let $\alpha \in M_{N}(k)$ be an $N \times N$ nilpotent matrix with coefficients in $k$. The Jordan type of $\alpha$ is the partition of $N, \underline{n}=n_{1} \geq n_{2} \geq \cdots \geq n_{s}$ with $\sum_{j} n_{j}=N$, such that the canonical Jordan form of $\alpha$ is a direct sum of indecomposable blocks of size $n_{j}$.

If $\alpha, \beta \in M_{N}(k)$ are two nilpotent matrices, we write $\alpha \geq \beta$ (and say that the Jordan type of $\alpha$ is greater or equal to the Jordan type of $\beta$ ) if the partition associated to $\alpha$ is greater or equal to the partition associated to $\beta$. The condition that $\alpha \geq \beta$ is equivalent to the condition that

$$
\operatorname{rank}\left(\alpha^{s}\right) \geq \operatorname{rank}\left(\beta^{s}\right), \quad \forall s>0,
$$

(see $[7,6.2 .2]$ ) which in turn is equivalent to the condition that

$$
\operatorname{dim}_{k} \operatorname{Ker}\left(\alpha^{s}\right) \leq \operatorname{dim}_{k} \operatorname{Ker}\left(\beta^{s}\right), \quad \forall s>0 .
$$

We denote by $\sim$ the equivalence relation associated to this partial ordering:

$$
\alpha \sim \beta \quad \text { iff } \quad \alpha \leq \beta \text { and } \beta \leq \alpha .
$$

Observe that $\alpha \sim \beta$ if and only if $\alpha$ and $\beta$ have the same Jordan type if and only if they are conjugate (under the adjoint action of $G L_{N}(k)$ ) elements of $M_{N}(k)$.

The data of the Jordan type of a nilpotent matrix $\alpha \in M_{N}(k)$ is equivalent to each of the following:

- the isomorphism class of $k^{N}$ as a $k[t]$-module, with $t$ acting as $\alpha$;

- the data of the ranks of $\alpha^{s}, \forall s>0$;

- the data of the dimensions of the kernels of $\alpha^{s}, \forall s>0$.

If $M$ is an $N$-dimensional $k[t]$-module with $t$ acting nilpotently, then we shall frequently refer to the "Jordan type of $M$ " rather than the Jordan type of the matrix in $M_{N}(k)$ given by the action of $t$.

Lemma 1.2. Let $\alpha, \alpha_{1}, \cdots, \alpha_{n} \in M_{N}(k)$ be a family of commuting nilpotent matrices with coefficients in an infinite field $k$. The following conditions are equivalent:

(1) For all $\lambda_{1}, \cdots, \lambda_{n} \in k$,

$$
\operatorname{rank}(\alpha) \geq \operatorname{rank}\left(\alpha+\lambda_{1} \alpha_{1}+\cdots+\lambda_{n} \alpha_{n}\right)
$$

where $\alpha, \alpha+\lambda_{1} \alpha_{1}+\cdots+\lambda_{n} \alpha_{n}$ are viewed as matrices in $M_{N}(k)$. 
(2) For every field extension $K / k$, all $\lambda_{1}, \cdots, \lambda_{n} \in K$,

$$
\operatorname{rank}(\alpha) \geq \operatorname{rank}\left(\alpha+\lambda_{1} \alpha_{1}+\cdots+\lambda_{n} \alpha_{n}\right)
$$

where $\alpha, \alpha+\lambda_{1} \alpha_{1}+\cdots+\lambda_{n} \alpha_{n}$ are viewed as matrices in $M_{N}(K)$.

(3) For $t_{1}, \ldots, t_{n}$ independent variables in the field $k\left(t_{1}, \ldots, t_{n}\right)$ of rational functions in $n$ variables over $k$,

$$
\operatorname{rank}(\alpha)=\operatorname{rank}\left(\alpha+t_{1} \alpha_{1}+\cdots+t_{n} \alpha_{n}\right) .
$$

Proof. For any $A \in M_{N}(k)$ and any pair of subsets $I, J \subset\{1, \ldots, N\}$ of cardinality $s \leq N$, denote by $A_{I, J}$ the associated minor, an $s \times s$ submatrix of $A$. The condition $\operatorname{rank} A \geq s$ is equivalent to the condition that there exist $I$ and $J$ such that $\operatorname{det}\left(\mathcal{A}_{I, J}\right) \neq 0$. For any $I$ and $J$ as above, consider

$$
d_{I, J}\left(t_{1}, \ldots, t_{n}\right)=\operatorname{det}\left(\left(\alpha+t_{1} \alpha_{1}+\cdots+t_{n} \alpha_{n}\right)_{I, J}\right)
$$

where $t_{1}, \ldots, t_{n}$ are independent variables in the field $k\left(t_{1}, \ldots, t_{n}\right)$. Let $K / k$ be an arbitrary field extension. Then $d_{I, J}\left(t_{1}, \ldots, t_{n}\right)=0$ if and only if $d_{I, J}\left(\lambda_{1}, \ldots, \lambda_{n}\right)=$ 0 for all $\left(\lambda_{1}, \ldots, \lambda_{n}\right)$ in an open dense subset in $\mathbb{A}_{K}^{n}$. Thus,

$\operatorname{rank}\left(\alpha+t_{1} \alpha_{1}+\cdots+t_{n} \alpha_{n}\right) \geq \operatorname{rank}\left(\alpha+\lambda_{1} \alpha_{1}+\cdots+\lambda_{n} \alpha_{n}\right)$ for all $\lambda_{1}, \ldots, \lambda_{n} \in K$, $\operatorname{rank}\left(\alpha+t_{1} \alpha_{1}+\cdots+t_{n} \alpha_{n}\right)=\operatorname{rank}\left(\alpha+\lambda_{1} \alpha_{1}+\cdots+\lambda_{n} \alpha_{n}\right)$ for all $\left(\lambda_{1}, \ldots, \lambda_{n}\right)$ in an open dense subset in $\mathbb{A}_{K}^{n}$.

Since the argument of the proof of Lemma 1.2 applies equally to all powers of $\alpha$, we immediately conclude the following proposition.

Proposition 1.3. Let $\alpha, \alpha_{1}, \ldots, \alpha_{n} \in M_{N}(k)$ be a family of commuting nilpotent matrices with coefficients in an infinite field $k$. Then the following conditions are equivalent:

(1) For all $\lambda_{1}, \ldots, \lambda_{n} \in k$,

$$
\alpha \geq \alpha+\lambda_{1} \alpha_{1}+\cdots+\lambda_{n} \alpha_{n} .
$$

(2) For every field extensions $K / k$, all $\lambda_{1}, \ldots, \lambda_{n} \in K$,

$$
\alpha \geq \alpha+\lambda_{1} \alpha_{1}+\cdots+\lambda_{n} \alpha_{n} .
$$

(3) For $t_{1}, \ldots, t_{n}$ independent variables in the field $k\left(t_{1}, \ldots, t_{n}\right)$ of rational functions in $n$ variables over $k$,

$$
\alpha \sim \alpha+t_{1} \alpha_{1}+\cdots+t_{n} \alpha_{n}
$$

in $M_{N}\left(k\left(t_{1}, \ldots, t_{n}\right)\right)$.

Definition 1.4. We say that $\alpha$ is rank maximal with respect to $\alpha_{1}, \ldots, \alpha_{n}$ provided that the equivalent conditions of Lemma 1.2 are satisfied for $\alpha, \alpha_{1}, \ldots, \alpha_{n} \in M_{N}(k)$.

Similarly, we say that $\alpha$ is maximal with respect to $\alpha_{1}, \ldots, \alpha_{n}$ if the equivalent conditions of Proposition 1.3 are satisfied for $\alpha, \alpha_{1}, \ldots, \alpha_{n} \in M_{N}(k)$.

Remark 1.5. Let $\alpha_{1}, \ldots, \alpha_{n} \in M_{N}(k)$ be a family of nilpotent commuting matrices with coefficients in an infinite field $k$. There exists $\alpha \in M_{N}(k)$ satisfying the conditions of Proposition 1.3. Indeed, let $s_{1}, \ldots, s_{n}$ be a set of indeterminants. Let

$$
\alpha_{\underline{s}}=s_{1} \alpha_{1}+\cdots+s_{n} \alpha_{n} \in M_{N}\left(k\left(s_{1}, \ldots, s_{n}\right)\right) .
$$

Observe that $\alpha_{\underline{s}}=s_{1} \alpha_{1}+\cdots+s_{n} \alpha_{n} \sim\left(s_{1}+t_{1}\right) \alpha_{1}+\cdots+\left(s_{n}+t_{n}\right) \alpha_{n}=\alpha_{\underline{s}}+t_{1} \alpha_{1}+\cdots+$ $t_{n} \alpha_{n}$ as elements of $M_{N}\left(k\left(s_{1}, \ldots, s_{n}, t_{1}, \ldots, t_{n}\right)\right)$. Indeed, $\left(s_{1}+t_{1}\right) \alpha_{1}+\cdots+\left(s_{n}+\right.$ 
$\left.t_{n}\right) \alpha_{n}$ can be specialized to $s_{1} \alpha_{1}+\cdots+s_{n} \alpha_{n}$ by setting $t_{i}=0$. On the other hand, if one makes a change of variables $s_{i}^{\prime}=s_{i}+t_{i}$, then $\alpha_{s}=\left(s_{1}^{\prime}-t_{1}\right) \alpha_{1}+\cdots+\left(s_{n}^{\prime}-t_{n}\right) \alpha_{n}$ can be specialized to $\alpha_{\underline{s}}+t_{1} \alpha_{1}+\cdots+t_{n} \alpha_{n}=s_{1}^{\prime} \alpha_{1}+\cdots+s_{n}^{\prime} \alpha_{n}$ by taking $t_{i}=0$. Thus, $\alpha_{\underline{s}}$ has the same Jordan type as $\alpha_{\underline{s}}+t_{1} \alpha_{1}+\cdots+t_{n} \alpha_{n}$. Proposition 1.3 implies that $\alpha_{\underline{s}}$ is maximal with respect to $\alpha_{1}, \ldots, \alpha_{n}$.

By upper semi-continuity of the Jordan type, there exists an open dense subset in $\mathbb{A}_{k}^{n}$ such that for any $\underline{\lambda}=\left(\lambda_{1}, \ldots, \lambda_{n}\right)$ from that subset we have $\alpha_{\underline{\lambda}}=\lambda_{1} \alpha_{1}+$ $\cdots+\lambda_{n} \alpha_{n} \sim \alpha_{\underline{s}}$. For any such $\underline{\lambda}, \alpha=\alpha_{\underline{\lambda}}$ satisfies the conditions of Proposition 1.3 .

We proceed to investigate the rank maximality condition in the special case of two commuting matrices. For any commuting nilpotent matrices $\alpha, \beta \in M_{N}(k)$ set

$$
W_{\alpha, \beta}=\sum_{\mu \in k^{*}} \operatorname{Ker}(\alpha+\mu \beta) \subset k^{m},
$$

the subspace spanned by the union of the subspaces $\operatorname{Ker}(\alpha+\mu \beta)$. Since $\alpha, \beta$ commute, each $\operatorname{Ker}(\alpha+\mu \beta)$ is invariant under both $\alpha$ and $\beta$.

Lemma 1.6. Let $\alpha, \beta \in M_{N}(k)$ be a pair of commuting matrices and set $W=$ $W_{\alpha, \beta}$. Then $\beta(W)=\alpha(W) \subset W$ and hence

$$
(\alpha+\lambda \beta)(W) \subset \alpha(W) \quad \text { for all } \lambda \in k .
$$

Proof. For any $\mu \in k^{*}$, we have $\left.\beta\right|_{\operatorname{Ker}(\alpha+\mu \beta)}=-\left.\mu^{-1} \alpha\right|_{\operatorname{Ker}(\alpha+\mu \beta)}$. Hence $\beta(\operatorname{Ker}(\alpha+$ $\mu \beta))=\alpha(\operatorname{Ker}(\alpha+\mu \beta))$. Thus $\beta(W)=\alpha(W)$. The second claim is now obvious.

Using Lemma 1.6, we obtain a useful criterion for when $\alpha$ is rank maximal with respect to $\alpha, \beta$.

Lemma 1.7. Let $k$ be an infinite field, let $\alpha, \beta \in M_{N}(k)$ be two commuting nilpotent matrices, and set $W=W_{\alpha, \beta}$. Then the following conditions are equivalent

- $\alpha$ is rank maximal with respect to $\beta$.

- $\operatorname{Ker}(\alpha) \subset W$.

Proof. Set $r=\max _{\lambda \in k} \operatorname{rank}(\alpha+\lambda \beta)$. As seen in the proof of Lemma 1.2, for all but finitely many $\lambda \in k$ we have an equality $\operatorname{rank}(\alpha+\lambda \beta)=r$. Hence, $\alpha$ is rank maximal with respect to $\beta$ if and only if $\operatorname{rank}(\alpha)=r$, that is $\operatorname{dim}(\operatorname{Ker}(\alpha))=N-r$. By Lemma 1.6, $\operatorname{rank}\left(\left.\alpha\right|_{W}\right) \geq \operatorname{rank}\left(\left.(\alpha+\lambda \beta)\right|_{W}\right)$ for all $\lambda \in k$ with equality holding for all but finitely many $\lambda$ 's. Thus for a "generic" $\lambda \in k$, we have the following chain of equalities and inequalities:

$$
\begin{aligned}
& \operatorname{dim}(\operatorname{Ker} \alpha) \geq \operatorname{dim}((\operatorname{Ker} \alpha) \cap W)=\operatorname{dim}\left(\operatorname{Ker}\left(\left.\alpha\right|_{W}\right)\right) \\
= & \operatorname{dim}\left(\operatorname{Ker}\left(\left.(\alpha+\lambda \beta)\right|_{W}\right)=\operatorname{dim}(\operatorname{Ker}(\alpha+\lambda \beta))=N-r\right.
\end{aligned}
$$

where the last but one equality holds since $\operatorname{Ker}(\alpha+\lambda \beta) \subset W$ for all $\lambda \in k^{*}$ by definition of $W$. This chain shows that $\operatorname{dim}(\operatorname{Ker} \alpha)=N-r$ if and only if Ker $\alpha \subset W$.

Lemma 1.7 will now enable us to prove the following key property of rank maximality. In some sense, the property proved in Proposition 1.8 is the essential case of the general maximality result of Theorem 1.12. 
Proposition 1.8. Let $k$ be an infinite field and let $\alpha, \beta \in M_{N}(k)$ be two commuting nilpotent matrices with $\alpha$ rank maximal with respect to $\beta$. Then for any nilpotent matrix $\gamma \in M_{N}(k)$ commuting with both $\alpha$ and $\beta$,

$$
\operatorname{rank}(\alpha)=\operatorname{rank}(\alpha+\beta \gamma)
$$

Proof. Set $W=W_{\alpha, \beta}$. Since $\gamma$ commutes with $\alpha$ and $\beta, W$ is $\gamma$-invariant.

We first verify that $\alpha(W)=(\alpha+\beta \gamma)(W)$. To prove this formula it suffices to show that $\alpha\left(V_{\mu}\right)=(\alpha+\beta \gamma)\left(V_{\mu}\right)$, where $\mu \in k^{*}$ and $V_{\mu}=\operatorname{Ker}(\alpha+\mu \beta)$. This follows immediately from the following relations:

$$
\left.\alpha\right|_{V_{\mu}}=-\left.\mu \cdot \beta\right|_{V_{\mu}},\left.\quad(\alpha+\beta \gamma)\right|_{V_{\mu}}=-\left.\mu \cdot \beta\right|_{V_{\mu}} \circ\left(1-\left.\mu^{-1} \cdot \gamma\right|_{V_{\mu}}\right)
$$

since the matrix $1-\left.\mu^{-1} \cdot \gamma\right|_{V_{\mu}}$ is invertible.

Next we show that $\operatorname{Ker}(\alpha+\beta \gamma) \subset W$. Let $v \in \operatorname{Ker}(\alpha+\beta \gamma)$ be an arbitrary vector in $k^{N}$. We are going to show that $\gamma^{i}(v) \in W$ for all $i \geq 0$ using descending induction on $i$. Since $\gamma$ is nilpotent, this statement is trivial for $i$ large enough. Assume now that $\gamma^{i+1}(v) \in W$. Then $\alpha\left(\gamma^{i}(v)\right)=-\beta \gamma^{i+1}(v)=\beta\left(-\gamma^{i+1}(v)\right) \in \beta(W)=\alpha(W)$. Thus there exists $w \in W$ such that $\alpha\left(\gamma^{i}(v)-w\right)=0$. Hence $\gamma^{i}(v) \in W+\operatorname{Ker} \alpha=W$, since Ker $\alpha \subset W$ according to Lemma 1.7.

Using $\alpha(W)=(\alpha+\beta \gamma)(W)$ and $\operatorname{Ker}(\alpha+\beta \gamma) \subset W$, we conclude that

$$
\begin{gathered}
\operatorname{rank}(\alpha+\beta \gamma)=N-\operatorname{dim}(\operatorname{Ker}(\alpha+\beta \gamma))=N-\operatorname{dim}\left(\operatorname{Ker}\left(\left.(\alpha+\beta \gamma)\right|_{W}\right)\right)= \\
=N-\operatorname{dim} W+\operatorname{dim}(\alpha+\beta \gamma)(W)=N-\operatorname{dim} W+\operatorname{dim} \alpha(W)= \\
=N-\left.\operatorname{dim} \operatorname{Ker} \alpha\right|_{W}=N-\operatorname{dim} \operatorname{Ker} \alpha=\operatorname{rank} \alpha
\end{gathered}
$$

The following theorem establishes that rank maximality with respect to linear combinations of $\alpha_{1}, \ldots, \alpha_{n}$ implies rank maximality with respect to all polynomials in $\alpha_{1}, \ldots, \alpha_{n}$. Our ultimate theorem, Theorem 1.12, refines this by replacing rank maximality by maximality.

Theorem 1.9. Let $\alpha, \alpha_{1}, \cdots, \alpha_{n}, \beta_{1}, \ldots, \beta_{n} \in M_{N}(k)$ be a family of commuting nilpotent matrices with coefficients in an infinite field $k$. Assume that $\alpha$ is rank maximal with respect to the family $\alpha_{1}, \ldots, \alpha_{n}$. Then $\alpha$ is also rank maximal with respect to the family $\alpha_{1}, \ldots, \alpha_{n}, \alpha_{1} \beta_{1}, \ldots, \alpha_{n} \beta_{n}$.

Moreover, $\operatorname{rank} \alpha=\operatorname{rank}\left(\alpha+\alpha_{1} \beta_{1}+\cdots+\alpha_{n} \beta_{n}\right)$.

Proof. Our procedure is to expand the family of matrices with respect to which $\alpha$ is rank maximal. that

Our first (and key) step is to show that we can add $\alpha_{1} \beta_{1}$. We proceed to verify

$$
\operatorname{rank} \alpha=\operatorname{rank}\left(\alpha+t_{1} \alpha_{1}+\cdots+t_{n} \alpha_{n}+t \alpha_{1} \beta_{1}\right)
$$

for independent variables $t_{1}, \ldots, t_{n}, t$. Observe that $\alpha+t_{1} \alpha_{1}+\ldots+t_{n} \alpha_{n}$ is obviously rank maximal with respect to $\alpha_{1}$, so that Proposition 1.8 thus implies that

$$
\operatorname{rank}\left(\alpha+t_{1} \alpha_{1}+\cdots+t_{n} \alpha_{n}+t \alpha_{1} \beta_{1}\right)=\operatorname{rank}\left(\alpha+t_{1} \alpha_{1}+\cdots+t_{n} \alpha_{n}\right) .
$$

On the other hand,

$$
\operatorname{rank}\left(\alpha+t_{1} \alpha_{1}+\cdots+t_{n} \alpha_{n}\right)=\operatorname{rank} \alpha
$$

in view of rank maximality of $\alpha$ with respect to $\alpha_{1}, \ldots, \alpha_{n}$. 
Iterating this procedure, we see that we can add all terms of the form $\alpha_{i} \beta_{i}$ to our family, thereby establishing the rank maximality of $\alpha$ with respect to the family $\alpha_{1}, \ldots, \alpha_{n}, \alpha_{1} \beta_{1}, \ldots, \alpha_{n} \beta_{n}$.

To prove the rank maximality of $\alpha+\alpha_{1} \beta_{1}+\cdots+\alpha_{n} \beta_{n}$ and the equality rank $\alpha=$ $\operatorname{rank}\left(\alpha+\alpha_{1} \beta_{1}+\cdots+\alpha_{n} \beta_{n}\right)$, we first verify these assertions with $\alpha_{1} \beta_{1}$. The equality of ranks follows immediately from Proposition 1.8. There remains to show that for independent variables $t_{1}, \ldots, t_{n}$ we have the equality

$$
\operatorname{rank}\left(\alpha+\alpha_{1} \beta_{1}\right)=\operatorname{rank}\left(\alpha+\alpha_{1} \beta_{1}+t_{1} \alpha_{1}+\cdots+t_{n} \alpha_{n}\right)
$$

Since $\alpha+t_{1} \alpha_{1}+\cdots+t_{n} \alpha_{n}$ is rank maximal with respect to $\alpha_{1}$, two applications of Proposition 1.8 imply the equalities

$$
\begin{gathered}
\operatorname{rank}\left(\alpha+t_{1} \alpha_{1}+\cdots+t_{n} \alpha_{n}+\alpha_{1} \beta_{1}\right)=\operatorname{rank}\left(\alpha+t_{1} \alpha_{1}+\cdots+t_{n} \alpha_{n}\right)=\operatorname{rank} \alpha= \\
=\operatorname{rank}\left(\alpha+\alpha_{1} \beta_{1}\right) .
\end{gathered}
$$

Finally, we get the general case by iterating this procedure.

To extend Theorem 1.9 to establish maximality of Jordan types, we employ the following auxilliary construction which reduces maximality to rank maximality of associated matrices. Denote by $J_{s} \in M_{s}(k)$ the Jordan block of size $s$, i.e. $J_{s}$ is a nilpotent matrix which acts on the standard basis of $k^{s}$ according to the rule $J_{s}\left(e_{1}\right)=0, J_{s}\left(e_{i}\right)=e_{i-1} \quad(2 \leq i \leq s)$.

Lemma 1.10. For any finite dimensional $k$-vector space $V$ and any $\alpha \in \operatorname{End}_{k}(V)$, there is a natural isomorphism

$$
\operatorname{Ker}\left(\alpha^{s}\right) \simeq \operatorname{Ker}\left(\alpha \otimes 1_{s}+1_{V} \otimes J_{s}\right) .
$$

where $1_{s}, 1_{V}$ are identity operators on $k^{s}, V$ respectively, and $\alpha \otimes 1_{s}+1_{V} \otimes J_{s} \in$ $\operatorname{End}_{k}\left(V \otimes k^{s}\right)$.

Proof. Any $v \in V \otimes k^{s}$ can be written uniquely in the form $v_{1} \otimes e_{1}+\cdots+v_{s} \otimes e_{s}$ with appropriate $v_{1}, \ldots, v_{s} \in V$. Moreover

$$
\left(\alpha \otimes 1_{s}+1_{V} \otimes J_{s}\right)\left(v_{1} \otimes e_{1}+\cdots+v_{s} \otimes e_{s}\right)=\sum_{i=1}^{s-1}\left(\alpha\left(v_{i}\right)+v_{i+1}\right) \otimes e_{i}+\alpha\left(v_{s}\right) \otimes e_{s}
$$

Thus, equations defining $\operatorname{Ker}\left(\alpha \otimes 1_{s}+1_{V} \otimes J_{s}\right)$ look as follows:

$$
\alpha\left(v_{1}\right)=-v_{2}, \alpha\left(v_{2}\right)=-v_{3}, \ldots, \alpha\left(v_{s-1}\right)=-v_{s}, \alpha\left(v_{s}\right)=0 .
$$

We conclude that $v_{1}$ determines all other components via the formulae

$$
v_{i}=(-1)^{i-1} \alpha^{i-1}\left(v_{1}\right)
$$

and the only equation for $v_{1}$ is $\alpha^{s}\left(v_{1}\right)=0$, i.e. $v_{1} \in \operatorname{Ker} \alpha^{s}$.

Corollary 1.11. Let $\alpha, \alpha_{1}, \ldots, \alpha_{n} \in M_{N}(k)$ be a family of commuting nilpotent matrices with coefficients in an infinite field $k$. Then the following conditions are equivalent.

- $\operatorname{rank}\left(\alpha^{s}\right) \geq \operatorname{rank}\left(\alpha+\lambda_{1} \alpha_{1}+\cdots+\lambda_{n} \alpha_{n}\right)^{s} \quad \forall \lambda_{1}, \ldots, \lambda_{n} \in k$

- $\alpha \otimes 1_{s}+1_{N} \otimes J_{s}$ is rank maximal with respect to $\alpha_{1} \otimes 1_{s}, \ldots, \alpha_{n} \otimes 1_{s}$

We now formulate the main theorem of this section, a result which will play a key role in later sections in our consideration of "local maximality" for finite dimensional modules for an arbitrary finite group scheme. 
Theorem 1.12. Let $\alpha, \alpha_{1}, \ldots, \alpha_{n}, \beta_{1}, \ldots \beta_{n} \in M_{N}(k)$ be a finite set of commuting nilpotent matrices with coefficients in an infinite field $k$. Assume that $\alpha$ is maximal with respect to the family $\alpha_{1}, \ldots, \alpha_{n}$. Then $\alpha$ is also maximal with respect to the family $\alpha_{1}, \ldots, \alpha_{n}, \alpha_{1} \beta_{1}, \ldots, \alpha_{n} \beta_{n}$.

Moreover,

$$
\alpha \sim \alpha+\alpha_{1} \beta_{1}+\cdots+\alpha_{n} \beta_{n}
$$

Proof. This follows immediately by combining Theorem 1.9 and Corollary 1.11.

By taking $\beta_{i}$ to be polynomials in $\alpha_{j}$ without constant term, we obtain the following special case of Theorem 1.12.

Corollary 1.13. Assume that $\alpha$ is maximal with respect to a family $\alpha_{1}, \ldots, \alpha_{n} \in$ $M_{N}(k)$ of commuting nilpotent matrices. Then

$$
\alpha \geq \alpha+q\left(\alpha_{1}, \ldots, \alpha_{n}\right)
$$

for any polynomial $q \in k\left[X_{1}, \ldots, X_{n}\right]$ with no non-zero constant term.

Moreover if $q \in k\left[X_{1}, \ldots, X_{n}\right]$ with no non-zero constant or linear term, then

$$
\alpha \sim \alpha+q\left(\alpha_{1}, \ldots, \alpha_{n}\right) .
$$

Remark 1.14. A special case of Corollary 1.13 follows from the work of George McNinch [17] via the theory of nilpotent orbits. Namely, one needs to make an assumption that the Jordan type of all the matrices involved does not have blocks of size $p$. We thank George McNinch for his interest in our problem.

Remark 1.15. In a few degenerate cases considered below, we shall (implicitly) have to include the possibility that $\alpha, \alpha_{1}, \ldots, \alpha_{n} \in M_{N}(k)$ with $k$ finite. Then we shall say $\alpha$ is maximal with respect to $\alpha_{1}, \ldots, \alpha_{n}$ provided that this is the case when viewing these matrices in $M_{N}(K)$ for some infinite field extension $K / k$.

\section{MAXimal JoRdAn TYPES FOR ABELIAN Finite GROUP SCHEMES}

If $E=(\mathbb{Z} / p \mathbb{Z})^{\times r}$ is an elementary abelian $p$-group of rank $r$, then a choice of generators $g_{1}, \ldots, g_{r} \in E$ determines an isomorphism $k E \simeq k\left[t_{1}, \ldots, t_{r}\right] /\left(t_{1}^{p}, \ldots, t_{r}^{p}\right)$ given by sending $g_{i}$ to $t_{i}+1$. A cyclic shifted subgroup of $E$ with respect to the set of generators $\left\{g_{1}, \ldots, g_{r}\right\}$ is a map of $k$-algebras specified by some $\underline{\lambda}=\left(\lambda_{1}, \ldots, \lambda_{r}\right) \in$ $k^{r}$

$$
\alpha_{\underline{\lambda}}: k[t] / t^{p} \rightarrow k\left[t_{1}, \ldots, t_{r}\right] /\left(t_{1}^{p}, \ldots, t_{r}^{p}\right), \quad t \mapsto \sum_{i=1}^{r} \lambda_{i} t_{i} .
$$

A theorem of $\mathrm{W}$. Wheeler [22] asserts that for any finite dimensional $k E$-module $M$, the subset of those $\underline{\lambda} \in k^{r}$ for which $\alpha_{\lambda}^{*}(M)$ is not of maximal Jordan type is Zariski closed in $\mathbb{A}^{r}$.

In this section, we use Theorem 1.12 to verify that this maximal Jordan type is an invariant of the $k E$-module $M$ independent of the choice of generators of $k E$. In particular, the intrinsic, basis-free nature of this maximal Jordan type tells us that this Jordan type is also maximal if we view the action of $k E$ on $M$ as the action of the restricted enveloping algebra $u(\mathcal{E})$ of a rank $r$ abelian Lie algebra $\mathcal{E}$ with trivial $p$-restriction determined by an arbitrary choice of $k$-algebra isomorphism of $k E \simeq k \mathbb{G}_{a(1)}^{\times r}=u(\mathcal{E})$.

In Theorem 2.9, this intrinsic maximal Jordan type is shown to extend to the data of an arbitrary finite abelian group scheme over $k$ acting upon a finite dimensional $k$-vector space. 
In [10] (as corrected in [11]) and [12], the first two authors consider " $\pi$-points", an alternative to shifted subgroups of $E$ that generalizes to any finite group scheme. We recall this alternative in the following definition.

Definition 2.1. Let $G$ be a finite group scheme over $k$ with group algebra $k G$. A $\pi$-point of $G$ is a (left) flat map of $K$-algebras

$$
\alpha_{K}: K[t] / t^{p} \rightarrow K G
$$

which factors through the group algebra $K C_{K} \subset K G$ of some unipotent abelian subgroup scheme $C_{K} \subset G_{K}$ defined over some field extension $K / k$.

A $\pi$-point $\alpha_{K}$ is said to specialize to the $\pi$-point $\beta_{L}$ if for every finite dimensional $k G$-module $M$ the condition that $\beta_{L}^{*}\left(M_{L}\right)$ is free as an $L[t] / t^{p}$-module implies that $\alpha_{K}^{*}\left(M_{K}\right)$ is free as a $K[t] / t^{p}$-module.

If $\alpha_{K}$ specializes to $\beta_{L}$ and $\beta_{L}$ specializes to $\alpha_{K}$, then we say that $\alpha_{K}$ is equivalent to $\beta_{L}$ (denoted by $\alpha_{K} \sim \beta_{L}$ ). The equivalence class of the $\pi$-point $\alpha_{K}$ is denoted $\left[\alpha_{K}\right]$.

One of the fundamental properties of $\pi$-points is that there is a natural homeomorphism

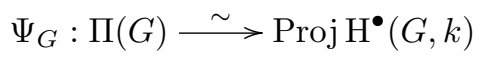

from the space $\Pi(G)$ of equivalence classes of $\pi$-points of $G$ (with topology intrinsi-

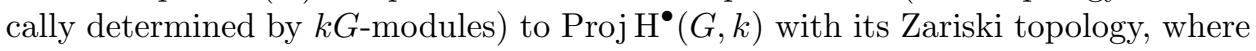
$\mathrm{H}^{\bullet}(G, k)$ is the commutative algebra of even dimensional cohomology of $G$ for $p$ odd and the commutative algebra of all cohomology of $G$ for $p=2$.

The following proposition makes explicit the equivalence relation $\alpha_{K} \sim \beta_{L}$ on $\pi$-points of an elementary abelian $p$-group $E$.

Proposition 2.2. Let $E=(\mathbb{Z} / p \mathbb{Z})^{\times r}$ be an elementary abelian $p$-group viewed as a finite group scheme over $k$. Choose some $k$-algebra isomorphism

$$
k E \simeq k\left[t_{1}, \ldots, t_{r}\right] /\left(t_{1}^{p}, \ldots, t_{r}^{p}\right) .
$$

Let $\alpha_{K}: K[t] / t^{p} \rightarrow K E, \beta_{L}: L[t] / t^{p} \rightarrow L E$ be two $\pi$-points of $k E$. Then $\alpha_{K} \sim \beta_{L}$ if and only if there exist embeddings $K \subset \Omega, L \subset \Omega$ over $k$ of $K, L$ into some field extension $\Omega$ over $k$ and some $0 \neq \omega \in \Omega$ such that

$$
\left(\alpha_{K} \otimes_{K} \Omega\right)(t)-\omega\left(\beta_{L} \otimes_{L} \Omega\right)(t) \in \Omega\left[t_{1}, \ldots, t_{r}\right] /\left(t_{1}^{p}, \ldots, t_{r}^{p}\right)
$$

is a polynomial in the $t_{i}$ 's with no non-zero constant or linear term.

Proof. The projectivized rank variety of $E$ can be identified with the projective space $\operatorname{Proj} I / I^{2}$ (see [2, II.4]) over an algebraically closed field $\Omega$ of characteristic $p$ where $I$ denotes the augmentation ideal of $\Omega E$. Since $\Omega$-rational points on the projectivized rank variety of $E$ are precisely equivalence classes of $\pi$-points defined over $\Omega([10,2.6,2.9])$, one concludes that two $\Omega$-rational $\pi$-points $\alpha, \beta: \Omega[t] / t^{p} \rightarrow$ $\Omega E$, are equivalent if and only if there exists is $0 \neq \omega \in \Omega$ such that $\alpha(t)-\omega \beta(t) \in$ $I^{2}$.

To prove the "if" direction, we observe that non-equivalent $\pi$-points remain non-equivalent after base change. Thus, the argument of the preceding paragraph implies that the asserted condition is sufficient to imply $\alpha_{K} \sim \beta_{L}$. 
Conversely, assume that $\alpha_{K}, \beta_{L}$ are equivalent as $\pi$-points of $k E$. Let $\Omega / k$ be an algebraically closed field containing both $K$ and $L$. Let $\sigma: \Omega \rightarrow \Omega$ be a field automorphism of $\Omega$ over $k$, and consider the action on $\pi$-points

$$
\sigma: \alpha_{\Omega} \mapsto \alpha_{\Omega}^{\sigma}
$$

given by sending $\alpha_{\Omega}$ to the $\Omega$-algebra map which sends $t$ to $\left(\alpha_{\Omega}(t)\right)^{\sigma}$. If $\rho: k G \rightarrow$ $\operatorname{End}_{k}(M)$ specifies a $k G$-module $M$, then $\rho\left(\alpha_{\Omega}^{\sigma}(t)\right)$ when viewed as a matrix is simply the result of applying $\sigma$ to the matrix entries of $\rho\left(\alpha_{\Omega}(t)\right)$.

The assertion that $\alpha_{\Omega}, \beta_{\Omega}$ are equivalent as $\pi$-points of $k E$ implies the existence of some automorphism $\sigma$ of $\Omega / k$ with the property that $\alpha_{\Omega}, \beta_{\Omega}^{\sigma}$ are equivalent as $\pi$-points of $\Omega E$. This is easily verified (as in $[12,4.6]$ ) by viewing equivalence classes of $\alpha_{\Omega}, \beta_{\Omega}$ as $\Omega$-rational points of $\operatorname{Proj} \mathrm{H}^{\bullet}(G, \Omega)$ which map to the same point in $\operatorname{Proj}^{\bullet}(G, k)$. Thus, another application of the argument in the first paragraph implies that we may find some $\omega \in \Omega$ such that $\alpha_{\Omega}(t)-\omega \cdot \beta_{\Omega}^{\sigma}(t) \in$ $\Omega\left[t_{1}, \ldots, t_{r}\right] /\left(t_{1}^{p}, \ldots, t_{r}^{p}\right)$ is a polynomial in the $t_{i}$ 's with no non-zero constant or linear term. Now twisting the embedding of $L \subset \Omega$ by the automorphism $\sigma$ we obtain the desired relationship between $\alpha_{K}$ and $\beta_{L}$.

The following example reflects the "typical" situation in which equivalent $\pi$ points $\alpha, \beta$ of an elementary abelian group $E$ lead to non-isomorphic $k[t] / t^{p}$-modules $\alpha^{*}(M), \beta^{*}(M)$

Example 2.3. Assume $p>2$. Let $E=\mathbb{Z} / p \mathbb{Z} \times \mathbb{Z} / p \mathbb{Z}$ be an elementary abelian group of rank 2, choose an identification of $k E$ with $k[x, y] /\left(x^{p}, y^{p}\right)$, and let $M$ be the $k E$-module (with respect to this identification of $k E$ ) defined as the quotient $k[x, y] /\left(x^{2}-y, x^{p}\right)$ of $k E$. Let $\alpha: k[t] / t^{p} \rightarrow k[x, y] /\left(x^{p}, y^{p}\right)$ be defined by sending $t$ to $x^{2}-y$ and let $\beta: k[t] / t^{p} \rightarrow k[x, y] /\left(x^{p}, y^{p}\right)$ be defined by sending $t$ to $y$. One readily checks that the $k[t] / t^{p}$-module $\alpha^{*}(M)$ is trivial whereas $\beta^{*}(M)$ consists of two Jordan blocks of sizes $\frac{p+1}{2}$ and $\frac{p-1}{2}$. Then $\alpha \sim \beta$ by Proposition 2.2, but $\alpha^{*}(M)$ is not isomorphic to $\beta^{*}(M)^{2}$.

The observation that the Jordan type can change when one replaces a cyclic shifted subgroup by another within the same equivalence class was made earlier by $\mathrm{S}$. Kaptanoglu, who also observed that for $E=\mathbb{Z} / 2 \mathbb{Z} \times \mathbb{Z} / 2 \mathbb{Z}$ this phenomenon does not occur $([16])$.

A major conclusion of our work is that if $\alpha_{K}$ has "maximal Jordan type" on a finite dimensional $k G$-module $M$ as defined below, then $\beta_{L}^{*}\left(M_{K}\right)$ has the same Jordan type as $\alpha_{K}^{*}\left(M_{K}\right)$ whenever $\alpha_{K} \sim \beta_{L}$. We prove this for an arbitrary finite group scheme $G$, proceeding in incremental steps of increasing generality, beginning with the case of an elementary abelian $p$-group.

Let $M_{1}$ be a $K[t] / t^{p}$-module, and $M_{2}$ be $L[t] / t^{p}$-module where $L, K$ are field estensions of $k$. Assume that $\operatorname{dim}_{K} M_{1}=\operatorname{dim}_{L} M_{2}=N$. Let the action of $t$ on $M_{1}$ and $M_{2}$ be given by nilpotent matrices of Jordan types $\underline{n}_{1}$ and $\underline{n}_{2}$ respectively, where $\underline{n}_{1}, \underline{n}_{2}$ are partitions of $N$. We say that the Jordan type of $M_{1}$ is greater or equal than the Jordan type of $M_{2}$, also written as $M_{1} \geq M_{2}$, if $\underline{n}_{1} \geq \underline{n}_{2}$.

We shall frequently apply this convention to the situation where we compare the Jordan types of $\alpha_{K}^{*}\left(M_{K}\right)$ and $\beta_{L}^{*}\left(M_{L}\right)$ for two $\pi$-points $\alpha_{K}, \beta_{L}$ and a finite dimensional $k G$-module $M$. 
Definition 2.4. Let $G$ be a finite group scheme over $k$ and let $M$ be a $k G$-module of dimension $N$. We say that a partition $\underline{n}$ of $N$ is a maximal Jordan type for $M$ if there exists some $\pi$-point $\alpha_{K}: K[t] / t^{p} \rightarrow K G$ such that the Jordan type of $\alpha_{K}^{*}\left(M_{K}\right)$ equals $\underline{n}$ and if there does not exist a $\pi$-point $\beta_{L}: L[t] / t^{p} \rightarrow L G$ such that the Jordan type of $\beta_{L}^{*}\left(M_{L}\right)$ is strictly greater than $\underline{n}$. For any $\pi$-point $\alpha_{K}$ such that the Jordan type of $\alpha_{K}^{*}\left(M_{K}\right)$ equals $\underline{n}$, we say that $\alpha_{K}^{*}\left(M_{K}\right)$ has maximal Jordan type for $M$, that the Jordan type of $\alpha_{K}^{*}\left(M_{K}\right)$ is maximal for $M$, and that $\alpha_{K}$ has maximal Jordan type on $M$.

Furthermore, we say that a partition $\underline{n}$ of $N$ is the absolute maximal Jordan type for $M$ if there exists some $\pi$-point $\alpha_{K}: K[t] / t^{p} \rightarrow K G$ such that the Jordan type of $\alpha_{K}^{*}\left(M_{K}\right)$ equals $\underline{n}$ and if $\underline{n}$ is greater or equal to the Jordan type of $\beta_{L}^{*}\left(M_{L}\right)$ for every $\pi$-point $\beta_{L}$ of $G$.

Remark 2.5. If $\Pi(G)$ is irreducible and if $\alpha_{K}$ is a generic $\pi$-point of $G$, then $\alpha_{K}^{*}\left(M_{K}\right)$ has absolute maximal Jordan type for $M$ by Corollary 4.11. On the other hand, we shall see in Example 4.13 that a $k G$-module can have more than one maximal Jordan type if $\Pi(G)$ is reducible.

Remark 2.6. Let $M$ be a $k G$-module and $\alpha_{K}: K[t] / t^{p} \rightarrow K G$ be a $\pi$-point of $G$ such that $\alpha_{K}^{*}\left(M_{K}\right)$ has maximal Jordan type for $M$. Let $i: C_{K} \subset G_{K}$ be a subgroup scheme such that $\alpha_{K}$ factors as $i \circ \alpha_{K}^{\prime}: K[t] / t^{p} \rightarrow K C_{K} \rightarrow K G$. Then $\alpha_{K}^{*}\left(M_{K}\right)=\alpha_{K}^{* *}\left(i^{*}\left(M_{K}\right)\right)$ has maximal Jordan type for the restriction $i^{*}\left(M_{K}\right)$ of $M_{K}$ to $C_{K}$.

The following theorem is a re-interpretation of Theorem 1.12 in terms of $\pi$-points of an elementary abelian $p$-group $E=(\mathbb{Z} / p \mathbb{Z})^{\times r}$ viewed as a finite group scheme over $k$. We say that a $\pi$-point of a finite group scheme $G$ is generic if its equivalence class is a generic point of $\Pi(G)$. One example of a generic $\pi$-point of $E=(\mathbb{Z} / p \mathbb{Z})^{\times r}$ is the map

$$
\nu_{k\left(x_{1}, \ldots, x_{r}\right)}: k\left(x_{1}, \ldots, x_{r}\right)[t] / t^{p} \rightarrow k\left(x_{1}, \ldots, x_{r}\right) E, \quad t \mapsto \sum_{i=1}^{r} x_{i} \cdot\left(g_{i}-1\right)
$$

where $\left\{g_{1}, \ldots, g_{r}\right\}$ is a set of generators for $E$.

Theorem 2.7. Let $E$ be an elementary abelian p-group of rank $r$, and let $\alpha_{K}$ : $K[t] / t^{p} \rightarrow K E$ be any generic $\pi$-point of $E$. Then for any finite dimensional $k E$ module $M$, the Jordan type of $\alpha_{K}^{*}\left(M_{K}\right)$ is the absolute maximal Jordan type for $M$.

Moreover, let $M$ be a finite dimensional $k E$-module and $\alpha_{K}$ be a $\pi$-point of $E$ such that the Jordan type of $\alpha_{K}^{*}\left(M_{K}\right)$ is the absolute maximal Jordan type for $M$. Then for any $\beta_{L} \sim \alpha_{K}$, the Jordan type of $\beta_{L}^{*}\left(M_{L}\right)$ equals that of $\alpha_{K}^{*}\left(M_{K}\right)$.

Proof. Let $\left\{g_{1}, \ldots, g_{r}\right\}$ be a set of generators for $E$, and let $\left\{t_{i}=g_{i}-1\right\}_{1 \leq i \leq r}$ be the corresponding polynomial generators of $k E$. Let $\rho: k G \rightarrow \operatorname{End}_{k}(M)$ specify the $k G$-module structure of $M$. According to Remark 1.5, the $\pi$-point $\nu_{k\left(x_{1}, \ldots, x_{r}\right)}$ of $(2.6 .1)$ has the property that $\rho\left(\nu_{k\left(x_{1}, \ldots, x_{r}\right)}(t)\right)$ is maximal with respect to the set $\left\{\rho\left(t_{1}\right), \ldots, \rho\left(t_{r}\right)\right\}$. Theorem 1.12 thus implies that the Jordan type of $\rho\left(\nu_{k\left(x_{1}, \ldots, x_{r}\right)}(t)\right)$ is greater or equal to the Jordan type of $\rho\left(\beta_{K}(t)\right)$ for any $\pi$-point $\beta_{K}: K[t] / t^{p} \rightarrow K G$. Thus, $\nu_{k\left(x_{1}, \ldots, x_{r}\right)}^{*}\left(M_{k\left(x_{1}, \ldots, x_{r}\right)}\right)$ has absolute maximal Jordan type for $M$. 
As mentioned above, $\nu_{k\left(x_{1}, \ldots, x_{r}\right)}$ is a generic $\pi$-point of $E$. Thus, to complete the proof of the theorem, it suffices to prove the second assertion. Let $M$ be a given finite dimensional $k E$-module $M$ with $k E$-structure specified by $\rho: k E \rightarrow$ $\operatorname{End}_{k}(M)$. Assume that $\alpha_{K}^{*}\left(M_{K}\right)$ has maximal Jordan type for $M$ so that $\rho\left(\alpha_{K}(t)\right)$ is maximal with respect to the set $\left\{\rho\left(t_{1}\right), \ldots, \rho\left(t_{r}\right)\right\}$. Since multiplying $\beta_{\Omega}$ by a scalar does not change the Jordan type of $\beta_{\Omega}^{*}\left(M_{\Omega}\right)$, Proposition 2.2 enables us to assume that $\alpha_{\Omega}(t)-\beta_{\Omega}(t) \in \Omega\left[t_{1}, \ldots, t_{r}\right] /\left(t_{1}^{p}, \ldots, t_{r}^{p}\right)$ is a polynomial in the $t_{i}$ 's with no non-zero constant or linear term for some field extension $\Omega / k$. Since extension of scalars preserves the "maximality" property by Proposition 1.3, we still have that $\rho\left(\alpha_{\Omega}(t)\right)$ is maximal with respect to the set $\left\{\rho\left(t_{1}\right), \ldots, \rho\left(t_{r}\right)\right\}$. Theorem 1.12 implies that $\rho\left(\alpha_{\Omega}(t)\right)$ has the same Jordan type as $\rho\left(\beta_{\Omega}(t)\right)$, thereby verifying that $\alpha_{\Omega}^{*}\left(M_{\Omega}\right)$ and $\beta_{\Omega}^{*}\left(M_{\Omega}\right)$ have the same Jordan type. Since extension of scalars preserves Jordan type, we conclude that $\alpha_{K}^{*}\left(M_{K}\right)$ and $\beta_{L}^{*}\left(M_{L}\right)$ have the same Jordan type.

The following lemma enables us to frequently replace $k$ by a conveniently chosen finite field extension $F / k$.

Lemma 2.8. Let $G$ be a finite group scheme defined over $k$, let $\alpha_{K}, \beta_{L}$ be $\pi$-points of $G$, and let $F / k$ be a finite normal extension. Then $\alpha_{K} \sim \beta_{L}$ as $\pi$-points of $G$ if and only if there exist embeddings of the composites $\widetilde{K}=F K, \widetilde{L}=F L$ in some field extension $\Omega / F$ such that $\alpha_{\widetilde{K}} \otimes_{\widetilde{K}} \Omega, \beta_{\widetilde{L}} \otimes_{\widetilde{L}} \Omega$ are equivalent $\pi$-points of $G_{F}$.

Moreover, for any finite field extension $F / k$, we have that $\alpha_{K}$ is a generic $\pi$-point of $G$ if and only if $\alpha_{\widetilde{K}}$ is a generic $\pi$-point of $G_{F}$, where $\widetilde{K}$ is again a composite of $F$ and $K$.

Proof. Observe that $\alpha_{K}, \beta_{L}$ are equivalent $\pi$-points of $G$ if and only if their base changes $\alpha_{\widetilde{K}}: \tilde{K}[t] / t^{p} \rightarrow \widetilde{K} G, \beta_{\widetilde{L}}: \tilde{L}[t] / t^{p} \rightarrow \widetilde{L} G$ remain equivalent $\pi$-points of $G$. Thus, it suffices to assume that $\widetilde{K}=K, \widetilde{L}=L$, so that $F \subset K \cap L$.

We first consider the case in which $F / k$ is separable and thus Galois. In this case, $\mathrm{H}^{\bullet}(G, k) \rightarrow \mathrm{H}^{\bullet}(G, F)=\mathrm{H}^{\bullet}(G, k) \otimes_{k} F$ is a Galois map, so that

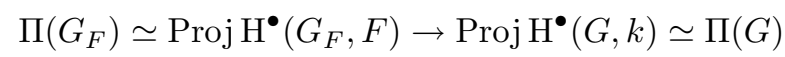

is a finite Galois covering. In particular, the pre-image of any generic point of $\Pi(G)$ is a generic point of $\Pi\left(G_{F}\right)$ and any two points in the pre-image of a given point of $\Pi(G)$ (such as $\left[\alpha_{K}\right]=\left[\beta_{L}\right]$ ) are conjugate by an element of $\operatorname{Gal}(F / k)$. Since the isomorphism $\Pi\left(G_{F}\right) \simeq \operatorname{Proj~} \mathrm{H}^{\bullet}\left(G_{F}, F\right)$ is compatible with the Galois action (see $[12,4.5])$, we get $\alpha_{K} \sim \beta_{L}$ as $\pi$-points of $G$ if and only if $\alpha_{\Omega} \sim \beta_{\Omega}^{\sigma}$ as $\pi$-points of $G_{F}$. Twisting the embedding of $L$ into $\Omega$ by $\sigma$, we obtain the desired result.

More generally, $F / k$ factors as $F / F^{s} / k$ where $F^{s} / k$ is separable and $F / F^{s}$ is purely inseparable. Now, the map $\mathrm{H}^{\bullet}\left(G, F^{s}\right) \rightarrow \mathrm{H}^{\bullet}(G, F)$ is a purely inseparable isogeny, so that

$$
\Pi\left(G_{F}\right) \simeq \operatorname{Proj~}^{\bullet}\left(G_{F}, F\right) \rightarrow \operatorname{Proj} H^{\bullet}\left(G_{F^{s}}, F^{s}\right) \simeq \Pi\left(G_{F^{s}}\right)
$$

is a bijection. Thus, the general case follows.

We now generalize Theorem 2.7 to an arbitrary finite abelian group scheme.

Theorem 2.9. Let $C$ be an abelian finite group scheme over $k$ and let $\alpha_{K}$ : $K[t] / t^{p} \rightarrow K C$ be any generic $\pi$-point of $C$. Then for any finite dimensional $k C$-module $M, \alpha_{K}^{*}\left(M_{K}\right)$ has absolute maximal Jordan type for $M$. 
Moreover, let $M$ be a finite dimensional $k C$-module and $\alpha_{K}$ be a $\pi$-point of $C$ such that $\alpha_{K}^{*}\left(M_{K}\right)$ has maximal Jordan type for $M$. Then for any $\beta_{L} \sim \alpha_{K}$, the Jordan type of $\beta_{L}^{*}\left(M_{L}\right)$ equals that of $\alpha_{K}^{*}\left(M_{K}\right)$.

Proof. Let $C^{D}$ be the Cartier dual of $C$. By [12, 4.2], we may find some finite extension $F / k$ such that $C_{F}^{D}=\left(C_{F}^{D}\right)^{0} \times \pi_{0}\left(C_{F}^{D}\right)$, where $\left(C_{F}^{D}\right)^{0}$ is the connected component of $C_{F}^{D}$. Dualizing again, we obtain

$$
C_{F}=\left(C_{F}^{D}\right)^{D}=\left(\left(C_{F}^{D}\right)^{0}\right)^{D} \times\left(\pi_{0}\left(C_{F}^{D}\right)\right)^{D} .
$$

The first factor is coconnected which is equivalent to unipotent, and the second factor is dual to an etale group scheme, and, hence, is semi-simple. Lemma 2.8 enables us to assume that $C$ itself is a product of a unipotent abelian finite group scheme and a semi-simple abelian finite group scheme.

Since any $\pi$-point of $C$ necessarily factors (by definition) through a unipotent abelian subgroup scheme of $C$, it must factor through $\left(\left(C^{D}\right)^{0}\right)^{D}$. Hence, we may assume that $C$ is itself unipotent. Let $\bar{k}$ be the perfect closure of $k$. By $[21,14.4]$, the group algebra $\bar{k} C$ is of the form $\bar{k}\left[t_{1}, \ldots, t_{r}\right] /\left(t_{1}^{p^{e_{1}}}, \ldots, t_{r}^{p^{e_{r}}}\right)$. Since every generator $t_{i}$ is defined over some finite subextension $k^{\prime} / k$, we can find a finite purely inseparable $L / k$ such that $L C=L\left[t_{1}, \ldots, t_{r}\right] /\left(t_{1}^{p^{e_{1}}}, \ldots, t_{r}^{p^{e_{r}}}\right)$. Hence, applying Lemma 2.8 again, we may assume

$$
k C \simeq k\left[t_{1}, \ldots, t_{r}\right] /\left(t_{1}^{p^{e_{1}}}, \ldots, t_{r}^{p^{e_{r}}}\right) .
$$

Let $T_{i}=t_{i}^{p^{e_{i}-1}}$. Any $\pi$-point $\alpha_{K}: K[t] / t^{p} \rightarrow K C$ must send $t$ to a polynomial in the $t_{i}$ 's, each monomial of which is divisible by some $T_{j}=t_{j}^{p^{e_{j}-1}}$ since $t^{p}=0$. As seen in Remark 1.5, the $\pi$-point

$$
\alpha_{k\left(x_{1}, \ldots, x_{r}\right)}: k\left(x_{1}, \ldots, x_{r}\right)[t] / t^{p} \rightarrow k\left(x_{1}, \ldots, x_{r}\right)\left[t_{1}, \ldots, t_{r}\right] /\left(t_{1}^{p^{e_{1}}}, \ldots, t_{r}^{p^{e_{r}}}\right)
$$

sending $t$ to $\sum_{i} x_{i} T_{i}$ is a generic $\pi$-point of $C$; moreover, $\rho\left(\alpha_{k\left(x_{1}, \ldots, x_{r}\right)}(t)\right)$ is maximal with respect to $\left\{\rho\left(T_{1}\right), \ldots, \rho\left(T_{r}\right)\right\}$ for any finite dimensional $k C$-module with structure specified by $\rho$ by Theorem 2.7. Thus, $\alpha_{k\left(x_{1}, \ldots, x_{r}\right)}^{*}\left(M_{k\left(x_{1}, \ldots, x_{r}\right)}\right)$ has absolute maximal Jordan type for any finite dimensional $k C$-module $M$ by Theorem 1.12. Moreover, if $M$ is a specified finite dimensional $k C$-module with structure specified by $\rho$ and if $\alpha_{K}^{*}\left(M_{K}\right)$ has absolute maximal Jordan type for $M$ and if $\beta_{L} \sim \alpha_{K}$, then as in the proof of Theorem 2.7 we conclude that Theorem 1.12 implies that $\alpha_{K}^{*}\left(M_{K}\right)$ and $\beta_{L}^{*}\left(M_{L}\right)$ have the same Jordan type.

\section{Generic And maximal Jordan types For infinitesimal group schemes}

Recall that a finite group scheme $G$ over $k$ is said to be infinitesimal if its coordinate algebra $k[G]$ is a (Artinian) local $k$-algebra. The height of such an infinitesimal group scheme is the least integer $r$ such that $f^{p^{r}}=0$ for all $f$ in the maximal ideal of $k[G]$. A 1-parameter subgroup of an infinitesimal group scheme $G$ of height $\leq r$ over some field extension $K / k$ is a morphism $\mathbb{G}_{a(r), K} \rightarrow G_{K}$ of finite group schemes over $K$. If $G$ is an infinitesimal group scheme of height $\leq r$, then the functor

$$
\begin{gathered}
\text { (f.g. commutative k-algebras) } \rightarrow \text { (sets) } \\
\qquad A \mapsto \operatorname{Hom}_{\text {grpsch } / A}\left(\mathbb{G}_{a(r), A}, G_{A}\right)
\end{gathered}
$$

is representable by an affine scheme denoted $V_{r}(G)=\operatorname{Spec} k\left[V_{r}(G)\right]([19,1.5])$. Here, $G_{A}$ is the group scheme over $A$ given by base change, $G_{A}=G \times_{\operatorname{Spec} k} \operatorname{Spec} A$. 
In particular, there is a universal 1-parameter subgroup

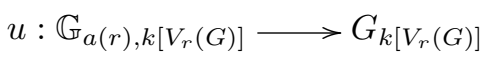

associated to the identity map of $k\left[V_{r}(G)\right]$ for any infinitesimal group scheme $G$. In some ways, the existence of this universal 1-parameter subgroup makes the study of representations of infinitesimal group schemes more tractable than the representations of finite groups.

In order to relate 1-parameter subgroups to $\pi$-points, we use the map of group algebras

$$
k \mathbb{G}_{a(1)}=k[t] / t^{p} \stackrel{t \mapsto u_{r-1}}{\longrightarrow} k\left[u_{0}, \ldots, u_{r-1}\right] /\left(u_{0}^{p}, \ldots, u_{r-1}^{p}\right)=k \mathbb{G}_{a(r)}
$$

where $u_{i}$ is the basis element dual to $t^{p^{i}}$ (so that $u_{i}=\frac{1}{p^{i} !} \frac{d^{p^{i}}}{d t^{p^{i}}}$ ). This is a map of Hopf algebras if and only if $r=1$ (in which case, we view this map as the identity). We employ the notation

$$
\epsilon: k \mathbb{G}_{a(1)} \longrightarrow k \mathbb{G}_{a(r)}
$$

for this map of group algebras, and stay alert to the fact that $\epsilon$ is not necessarily a map of Hopf algebras.

There is a natural map

$$
\widetilde{\Theta}_{G}: V_{r}(G) \backslash\{0\} \longrightarrow \Pi(G)
$$

which induces a homeomorphism (see $[20,5.2]$ and $[12,3.6]$ ) of Zariski topological spaces

$$
\Theta_{G}: \operatorname{Proj}\left(V_{r}(G)\right) \longrightarrow \Pi(G)
$$

This construction sends $x: \operatorname{Spec} k(x) \longrightarrow V_{r}(G)$ to

$$
\mu_{x} \circ \epsilon: k(x)[t] / t^{p} \longrightarrow k(x) \mathbb{G}_{a(r), k(x)} \longrightarrow k(x) G_{k(x)}=k(x) G
$$

where $\mu_{x}: k(x) \mathbb{G}_{a(r), k(x)} \longrightarrow k(x) G_{k(x)}$ is the map on group algebras induced by the 1-parameter subgroup $\mu_{x}$ associated to the point $x \in V_{r}(G)$ with residue field $k(x)$. Indeed, the composition $\Psi_{G} \circ \Theta_{G}$ of the homeomorphisms (2.1.1) and (3.0.2) is induced by the isogeny exhibited in [20, 5.2].

1-parameter subgroups give us "natural" representatives for equivalence classes of $\pi$-points. As we now verify, for each finite dimensional $k G$-module $M$ and each $\left[\alpha_{K}\right] \in \Pi(G)$, the Jordan type of $\alpha_{K}^{*}\left(M_{K}\right)$ is independent of the 1-parameter subgroup representing $\left[\alpha_{K}\right]$.

Proposition 3.1. Let $G$ be an infinitesimal group scheme. For any $\pi$-point $\alpha_{K}$, let $x \in V_{r}(G) \backslash\{0\}$ project onto $\left[\alpha_{K}\right] \in \Pi(G)$. Then

$$
\mu_{x} \circ \epsilon: k(x)[t] / t^{p} \rightarrow k(x) G \sim \alpha_{K}: K[t] / t^{p} \rightarrow K G .
$$

Moreover, if $\mu: \mathbb{G}_{a(r), K} \rightarrow G_{K}, \nu: \mathbb{G}_{a(r), L} \rightarrow G_{L}$ are non-zero 1-parameter subgroups of $G$ which map to the same equivalence class of $\pi$-points in $\Pi(G)$, then the Jordan type of $(\mu \circ \epsilon)^{*}\left(M_{K}\right)$ is the same as the Jordan type of $(\nu \circ \epsilon)^{*}\left(M_{L}\right)$.

Proof. The first assertion is merely a recollection of the definition of $\Theta_{G}$, granted that $\Theta_{G}(x)=\left[\alpha_{K}\right]$.

If $\mu: \mathbb{G}_{a(r), K} \rightarrow G_{K}$ is a 1-parameter subgroup for some field extension $K / k$ and if $k(x)$ is the residue field at the point $x \in V_{r}(G)$ determined by the map 
Spec $K \rightarrow V_{r}(G)$ corresponding to $\mu$, then $\mu$ determines an extension $K / k(x)$ of fields over $k$ and

$$
\mu=\mu_{x} \times_{\operatorname{Spec} k(x)} \operatorname{Spec} K .
$$

In particular, $(\mu \circ \epsilon)^{*}\left(M_{K}\right)$ has the same Jordan type as $\left(\mu_{x} \circ \epsilon\right)^{*}\left(M_{k(x)}\right)$.

Assume now that $\mu: \mathbb{G}_{a(r), K} \rightarrow G_{K}, \nu: \mathbb{G}_{a(r), L} \rightarrow G_{L}$ are 1-parameter subgroups determining the same point of $\Pi(G)$. By the preceding paragraph, we may assume that $K=L$. Then $\mu, \nu$ are related by the action $V_{r}(G) \times \mathbb{A}^{1} \rightarrow V_{r}(G)$ reflected in the grading of the coordinate algebra $k\left[V_{r}(G)\right]$. As can be seen using $[20,1.12]$, the action of some $a \in \mathbb{A}^{1}(K)$ sends $\mu \circ \epsilon: K[t] / t^{p} \rightarrow K G$ to $a^{p^{r-1}}(\mu \circ \epsilon): K[t] / t^{p} \rightarrow K G$. Thus, $(\mu \circ \epsilon)^{*}\left(M_{K}\right)$ has the same Jordan type as $(\nu \circ \epsilon)^{*}\left(M_{K}\right)$.

We say that a point $\eta \in V_{r}(G)$ specializes to a point $\zeta \in V_{r}(G)$ if there exists a map $\psi: \operatorname{Spec} R \rightarrow V_{r}(G)$ where $R$ is a local, integral $k$-algebra whose field of fractions has image $\eta$ and whose residue field has image $\zeta$. More generally, if $\mu: \mathbb{G}_{a(r), K} \rightarrow G, \nu: \mathbb{G}_{a(r), L} \rightarrow G$ are 1-parameter subgroups obtained by base change from $\eta, \zeta$ and if $\eta$ specializes to $\zeta$, then we say $\mu$ specializes to $\nu$.

Proposition 3.2. Let $G$ be an infinitesimal group scheme. Assume that the 1parameter subgroup $\mu: \mathbb{G}_{a(r), K} \rightarrow G$ specializes to the 1-parameter subgroup $\nu$ : $\mathbb{G}_{a(r), L} \rightarrow G$. Then for any finite dimensional $k G$-module $M$, the Jordan type of $(\mu \circ \epsilon)^{*}\left(M_{K}\right)$ is greater or equal to the Jordan type of $(\nu \circ \epsilon)^{*}\left(M_{L}\right)$.

Proof. Let $\eta, \zeta \in V_{r}(G)$ be such that $\mu: \mathbb{G}_{a(r), K} \rightarrow K G, \nu: \mathbb{G}_{a(r), L} \rightarrow L G$ are obtained by base change from $\mu_{\eta}, \nu_{\zeta}$. Let $\psi: \operatorname{Spec} R \rightarrow V_{r}(G)$ be a morphism with $R$ a local, integral $k$-algebra whose field of fractions $\Omega$ has image $\eta$ and whose residue field $F$ has image $\zeta$. This corresponds to an $R$-group scheme homomorphism $\psi: \mathbb{G}_{a(r), R} \rightarrow G_{R}$. By Proposition 3.1, it suffices to consider the $R[t] / t^{p}$-module obtained as the pull-back of $M_{R}$ via $\psi \circ \epsilon$ and show that the Jordan form of the endomorphism $t$ on $M_{R} \otimes_{R} \Omega$ is greater or equal to the Jordan form of the endomorphism $t$ on $M_{R} \otimes_{R} F$. This in turn is equivalent to proving for all $i, 1 \leq i<p$, that the rank of $t^{i}$ on $M_{R} \otimes_{R} \Omega$ is greater or equal to the rank of $t^{i}$ on $M_{R} \otimes_{R} F$. This "upper-semicontinuity" property follows easily from Nakayama's Lemma.

A simple geometric argument shows that if $\eta \in V_{r}(G)$ projects onto $\left[\alpha_{K}\right], \zeta \in$ $V_{r}(G)$ projects onto $\left[\beta_{L}\right]$, and $\left[\beta_{L}\right]$ is a specialization of $\left[\alpha_{K}\right]$ as points of $\Pi(G)$, then $\eta$ specializes to $\zeta$ as points of $V_{r}(G)$ as formulated prior to Proposition 3.2. The following property of Jordan types is now an immediate corollary of Propositions 3.1 and 3.2 .

Corollary 3.3. Let $G$ be an infinitesimal group scheme, and consider a $\pi$-point $\alpha_{K}: K[t] / t^{p} \rightarrow K G$ specializing to the $\pi$-point $\beta_{L}: L[t] / t^{p} \rightarrow L G$. Let $\mu:$ $\mathbb{G}_{a(r), \Omega} \rightarrow G_{\Omega}, \nu: \mathbb{G}_{a(r), \Sigma} \rightarrow G_{\Sigma}$ be non-zero 1-parameter subgroups of $G$ which project to $\left[\alpha_{k}\right],\left[\beta_{L}\right] \in \Pi(G)$. Then, for any finite dimensional $k G$-module $M$, the Jordan type of $(\mu \circ \epsilon)^{*}\left(M_{\Omega}\right)$ is greater or equal to the Jordan type of $(\nu \circ \epsilon)^{*}\left(M_{\Sigma}\right)$

Using Theorem 2.9, we now establish the well-definedness of the generic Jordan type (i.e., independence of the choice of representing generic $\pi$-point) of a finite dimensional $k G$ module for any infinitesimal group scheme $G$. 
Theorem 3.4. Let $G$ be an infinitesimal group scheme over $k$ and let

$$
\alpha_{K}: K[t] / t^{p} \rightarrow K G, \quad \beta_{L}: L[t] / t^{p} \rightarrow L G
$$

be equivalent generic $\pi$-points of $G$. Then for any finite dimensional $k G$-module $M, \alpha_{K}^{*}\left(M_{K}\right)$ has the same Jordan type as $\beta_{L}^{*}\left(M_{L}\right)$.

Proof. Write $\alpha_{K}=i_{C} \circ \alpha_{K}^{\prime}: K[t] / t^{p} \stackrel{\alpha_{K}^{\prime}}{\longrightarrow} K C_{K} \stackrel{i_{C}}{\longrightarrow} K G$ where $C_{K} \subset G_{K}$ is an abelian subgroup scheme (which is necessarily unipotent). Choose a 1-parameter subgroup $\mu: \mathbb{G}_{a(r), K} \rightarrow C_{K}$ such that $[\mu \circ \epsilon]=\left[\alpha_{K}^{\prime}\right] \in \Pi\left(C_{K}\right)$ and let $\eta: \mathbb{G}_{a(r), \Omega} \rightarrow$ $C_{\Omega}$ be another 1-parameter subgroup such that $\eta \circ \epsilon$ is a generic $\pi$-point of $C_{K}$. Let $M$ be a $k G$-module. By Theorem $2.9,(\eta \circ \epsilon)^{*}\left(i_{C}^{*} M_{\Omega}\right)$ has maximal Jordan type among all $\gamma_{F}^{*}\left(i_{C}^{*} M_{F}\right)$ as $F / K$ ranges over all field extensions over $K$, and $\gamma_{F}$ ranges over all $\pi$-points of $C_{K}$. Since $i_{C} \circ \eta \circ \epsilon \sim i_{C} \circ \mu \circ \epsilon$ as $\pi$-points of $G$, Proposition 3.1 implies that $(\mu \circ \epsilon)^{*}\left(i_{C}^{*} M_{K}\right)$ has the same Jordan type as $(\eta \circ \epsilon)^{*}\left(i_{C}^{*} M_{\Omega}\right)$, and thus is maximal among all $\gamma_{F}^{*}\left(i_{C}^{*} M_{F}\right)$ as $\gamma_{F}$ ranges over all $\pi$-points of $C_{K}$. Since $\mu \circ \epsilon \sim$ $\alpha_{K}^{\prime}$ as $\pi$-points of $C_{K}$, Theorem 2.9 implies that the Jordan type of $\alpha_{K}^{*}\left(M_{K}\right)=$ $\alpha_{K}^{\prime}\left(i_{C}^{*} M_{K}\right)$ is also the same as the Jordan type of $(\mu \circ \epsilon)^{*}\left(i_{C}^{*} M_{K}\right)$.

Consider $\beta_{L}=i_{D} \circ \beta_{L}^{\prime}: L[t] / t^{p} \stackrel{\beta_{L}^{\prime}}{\longrightarrow} L D_{L} \stackrel{i_{D}}{\longrightarrow} L G$ equivalent to $\alpha_{K}$ (where $D_{L} \subset G_{L}$ is a unipotent abelian subgroup scheme) and let $\nu: \mathbb{G}_{a(r), L} \rightarrow G_{L}$ be a 1-parameter subgroup scheme such that $[\nu \circ \epsilon]=\left[\beta_{L}^{\prime}\right] \in \Pi\left(D_{L}\right)$. The preceding argument tells us that the Jordan type of $\beta_{L}^{*}\left(M_{L}\right)$ is the same as the Jordan type of $(\nu \circ \epsilon)^{*}\left(i_{D}^{*} M_{L}\right)$. On the other hand, Proposition 3.1 implies that the Jordan type of $(\mu \circ \epsilon)^{*}\left(i_{C}^{*} M_{K}\right)$ equals that of $(\nu \circ \epsilon)^{*}\left(i_{D}^{*}\left(M_{L}\right)\right.$ since $\mu \circ \epsilon \sim \alpha_{K} \sim \beta_{L} \sim \nu \circ \epsilon$.

As seen implicitly in the proof of Theorem 2.9, $\Pi(C)$ is irreducible if $C$ is an abelian finite group scheme. However, this is not true for a general finite group scheme, even in the case of a height 1 infinitesimal group scheme (i.e., a restricted Lie algebra). For example, the nullcone of a Borel subalgebra of a reductive Lie algebra is not necessarily irreducible for small primes (see $[5]$ ). If $\Pi(G)$ is not irreducible, then the Jordan type at a generic point need not be maximal, but Corollary 4.11 asserts that maximal Jordan types for a given finite dimensional module $M$ are realized at generic points.

Of course, maximal Jordan types can be realized at $\pi$-points which are not generic. We see in the following theorem that if the Jordan type for a given $k G$ module is maximal at some $\pi$-point then this Jordan type is independent of the representative of that $\pi$-point.

Theorem 3.5. Let $G$ be an infinitesimal group scheme over $k$ and $M$ a finite dimensional $k G$-module. Let $\beta_{L}: L[t] / t^{p} \rightarrow L G$ be a $\pi$-point of $G$ with the property that the Jordan type of $\beta_{L}^{*}\left(M_{L}\right)$ is maximal for $M$. Then for any $\pi$-point $\alpha_{K}$ : $K[t] / t^{p} \rightarrow K G$ which specializes to $\beta_{L}$, the Jordan type of $\alpha_{K}^{*}\left(M_{K}\right)$ equals the Jordan type of $\beta_{L}^{*}\left(M_{L}\right)$.

Proof. As in the proof of 3.4 we write $\alpha_{K}=i_{C} \circ \alpha_{K}^{\prime}: K[t] / t^{p} \stackrel{\alpha_{K}^{\prime}}{\longrightarrow} K C_{K} \stackrel{i_{C}}{\longrightarrow} K G$ and $\beta_{L}=i_{D} \circ \beta_{L}^{\prime}: L[t] / t^{p} \stackrel{\beta_{L}^{\prime}}{\longrightarrow} L D_{L} \stackrel{i_{D}}{\longrightarrow} L G$ with $C_{K} \subset G_{K}, D_{L} \subset G_{L}$ abelian unipotent subgroup schemes. Let $\mu: \mathbb{G}_{a(r), K} \rightarrow C_{K}, \nu: \mathbb{G}_{a(r), L} \rightarrow D_{L}$ be 1-

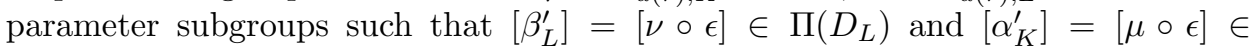


$\Pi\left(C_{K}\right)$. The maximality of $\beta_{L}^{*}\left(M_{L}\right)=\left(\beta_{L}^{\prime}\right)^{*}\left(i_{D}^{*}\left(M_{L}\right)\right)$ for $M$ and the fact that $\beta_{L}^{\prime} \sim \nu \circ \epsilon$ imply by Theorem 2.9 that the Jordan type of $\beta_{L}^{*}\left(M_{L}\right)$ equals that of $(\nu \circ \epsilon)^{*}\left(i_{D}^{*}\left(M_{L}\right)\right)=\left(\left(i_{D} \circ \nu\right) \circ \epsilon\right)^{*}\left(M_{L}\right)$. Because $\alpha_{K} \sim\left(i_{C} \circ \mu\right) \circ \epsilon$ specializes to $\beta_{L} \sim\left(i_{D} \circ \nu\right) \circ \epsilon$, Proposition 3.2 implies that $\left(\left(i_{C} \circ \mu\right) \circ \epsilon\right)^{*}\left(M_{K}\right)$ is likewise maximal for $M$. Thus, applying Theorem 2.9 to $\alpha_{K} \sim\left(i_{C} \circ \mu\right) \circ \epsilon$, we conclude that $\alpha_{K}^{*}\left(M_{K}\right)$ is also maximal for $M$.

\section{GENERIC AND MAXIMAL JORDAN TYPES FOR ARBITRARY FINITE GROUP SCHEMES}

Let $G$ be a finite group scheme of the form $G^{0} \rtimes \tau$, where the connected component $G^{0} \subset G$ is geometrically connected and where $\tau=\pi_{0}(G)$ is the (constant) group of connected components of $G$. For each elementary abelian $p$-subgroup $E \subset \tau$, define $\Pi_{0}\left(\left(G^{0}\right)^{E} \times E\right) \subset \Pi\left(\left(G^{0}\right)^{E} \times E\right)$ to be the subset of those $\pi$-points which do not admit a representative factoring through $\left(G^{0}\right)^{E} \times E^{\prime}$ with $E^{\prime}$ a proper subgroup of $E$. 4.13]):

Recall the "Quillen decomposition" for $\Pi(G)$ by locally closed subspaces ([12,

$$
\coprod \Pi_{0}\left(\left(G^{0}\right)^{E} \times E\right) / N_{\tau}(E) \simeq \Pi(G)
$$

where the disjoint union is indexed by conjugacy classes of elementary abelian $p$ subgroups of $\tau$, and where $N_{\tau}(E)$ denotes the normalizer of $E$ in $\tau$.

In order to consider the Jordan types $\alpha_{K}^{*}\left(M_{K}\right)$ for specific $\pi$-points representing $\left[\alpha_{K}\right] \in \Pi(G)$, we sharpen somewhat this decomposition by considering $\pi$-points up to the finer equivalence defining $\Pi(H)$ for subgroups $H$ of the form $\left(G^{0}\right)^{E} \times E$.

Proposition 4.1. Let $G$ be a finite group scheme of the form $G^{0} \rtimes \tau$, with $\tau=$ $\pi_{0}(G)$ constant and $G^{0}$ geometrically connected. Assume given $\left[\alpha_{K}\right] \in \Pi(G)$ and a representative

$$
\alpha_{K}=i \circ \alpha_{K}^{\prime}: K[t] / t^{p} \stackrel{\alpha_{K}^{\prime}}{\longrightarrow} K\left(\left(G^{0}\right)^{E} \times E\right) \stackrel{i}{\longrightarrow} K G
$$

of $\left[\alpha_{K}\right]$.

Then there exists a $\pi$-point $\alpha_{K}^{\prime \prime}: K[t] / t^{p} \rightarrow K\left(\left(G^{0}\right)^{E} \times E^{\prime}\right)$ equivalent to $\alpha_{K}^{\prime}$ as a $\pi$-point of $\left(G^{0}\right)^{E} \times E$ with $E^{\prime} \subset E$ such that $\left[\alpha_{K}\right] \in \Pi_{0}\left(\left(G^{0}\right)^{E^{\prime}} \times E^{\prime}\right)$.

Proof. Let $\alpha_{K}^{\prime}: K[t] / t^{p} \rightarrow K\left(\left(G^{0}\right)^{E} \times E\right)$ be given by

$$
t \mapsto \sum_{i=1}^{m} a_{i} f_{i}+\sum_{j=1}^{r} b_{j} s_{j}+p(\underline{f}, \underline{s}) \in K\left(G^{0}\right)^{E} \otimes_{K} K E,
$$

where $\left\{f_{i} ; 1 \leq i \leq n\right\}$ is a set of commuting nilpotent elements of $K\left(\left(G^{0}\right)^{E}\right)$, where $s_{j}=g_{j}-1$ with $\left\{g_{1}, \ldots, g_{r}\right\} \subset E$ a set of generators for $E$, where $a_{i}, b_{j}$ are elements of $K$, and where $p(f, \underline{s})$ is a polynomial in the $f_{i}, s_{j}$ without constant or linear term. Let $\alpha_{K, 0}^{\prime \prime}$ be the $\pi$-point of $G^{0}$ defined by

$$
\alpha_{K, 0}^{\prime \prime}(t)=\sum_{i=1}^{m} a_{i} f_{i}
$$


and $\alpha_{K, 1}^{\prime \prime}$ be the $\pi$-point of $\tau$ defined by

$$
\alpha_{K, 1}^{\prime \prime}(t)=\sum_{j=1}^{r} b_{j} s_{j} .
$$

Finally, let

$$
\alpha_{K}^{\prime \prime}=\alpha_{K, 0}^{\prime \prime}+\alpha_{K, 1}^{\prime \prime}
$$

and let $E^{\prime} \subset E$ equal the minimal subgroup such that $\sum_{j=1}^{r} b_{j} s_{j} \in K E^{\prime}$. By [10, 2.2] and a simple base change argument, $\alpha_{K}^{\prime \prime}$ is equivalent to $\alpha_{K}^{\prime}$ as a $\pi$-point of $\left(G^{0}\right)^{E} \times E$.

To complete the proof, it suffices to show that $\alpha_{K}$ is not equivalent (as a $\pi$-point of $G$ ) to some $\pi$-point factoring through $\left(G^{0}\right)^{E^{\prime \prime}} \times E^{\prime \prime}$ for some strictly smaller subgroup $E^{\prime \prime} \subset E^{\prime}$. This is immediate if $E^{\prime}$ is trivial. Thus, we may assume that $E^{\prime}$ is non-trivial.

Suppose there exists a $\pi$-point

$$
\gamma_{L}=i^{\prime} \circ \gamma_{L}^{\prime}: L[t] / t^{p} \stackrel{\gamma_{L}^{\prime}}{\longrightarrow} L\left(\left(G^{0}\right)^{E^{\prime \prime}} \times E^{\prime \prime}\right) \stackrel{i^{\prime}}{\longrightarrow} L G
$$

equivalent to $\alpha_{K}$ which factors through $\left(G^{0}\right)^{E^{\prime \prime}} \times E^{\prime \prime}$ with $E^{\prime \prime} \subset E^{\prime}$. We may assume that $E^{\prime \prime}$ is a minimal such subgroup for $\gamma_{L}$. By passing to a field extension if necessary, we may assume $L=K$. As for $\alpha_{K}$, let $\gamma_{K}^{\prime \prime}=\gamma_{K, 0}^{\prime \prime}+\gamma_{K, 1}^{\prime \prime}$ be the "linear" part of $\gamma_{K}^{\prime}$ with respect to the same set of generators $s_{i}$ of $K E$. If $N$ is a (finite dimensional) $\tau$-module, let $p^{*} N$ denote the $k G$-module with action given by projecting $k G$ to $k \tau$. Observe that for any finite-dimensional $k G$-module $M$, $\left(i \circ \alpha_{K}^{\prime \prime}\right)^{*}\left(M_{K}\right)$ is free if and only if $\left(i^{\prime} \circ \gamma_{K}^{\prime \prime}\right)^{*}\left(M_{K}\right)$ is free since $i \circ \alpha_{K}^{\prime \prime} \sim \alpha_{K} \sim \gamma_{K} \sim$ $i^{\prime} \circ \gamma_{K}^{\prime \prime}$. Since the action of $G^{0}$ on modules of the form $p^{*} N$ for a $\tau$-module $N$ is trivial, we conclude that $\left(i_{E} \circ \alpha_{K, 1}^{\prime \prime}\right)^{*}\left(N_{K}\right) \simeq\left(i \circ \alpha_{K}^{\prime \prime}\right)^{*}\left(p^{*} N_{K}\right)$, where $i_{E}: E \hookrightarrow \tau$ is the embedding of $E$ into $\tau$. Similarly, $\left(i_{E^{\prime \prime}} \circ \gamma_{K, 1}^{\prime \prime}\right)^{*}\left(N_{K}\right) \simeq\left(i \circ \gamma_{K}^{\prime \prime}\right)^{*}\left(p^{*} N_{K}\right)$, where $i_{E^{\prime \prime}}: E^{\prime \prime} \hookrightarrow \tau$ is the embedding of $E^{\prime \prime}$ into $\tau$. Since $N$ can be taken to be an arbitrary $\tau$-module, we conclude that $i_{E} \circ \alpha_{K, 1}^{\prime \prime} \sim i_{E^{\prime \prime}} \circ \gamma_{K, 1}^{\prime \prime}$ as $\pi$-points of $\tau$.

By the minimality assumption on $E^{\prime}$ for $\alpha_{K}^{\prime \prime}$, we conclude that $\left[\alpha_{K, 1}^{\prime \prime}\right] \in \Pi_{0}\left(E^{\prime}\right)$. Similarly, $\left[\gamma_{K, 1}^{\prime \prime}\right] \in \Pi_{0}\left(E^{\prime \prime}\right)$. If $E^{\prime \prime}$ were a proper subgroup of $E^{\prime}$, then the "Quillen decomposition" for the finite group $\tau$ (see (4.0.1)) would imply that $\Pi_{0}\left(E^{\prime}\right) / N_{\tau}\left(E^{\prime}\right) \cap$ $\Pi_{0}\left(E^{\prime \prime}\right) / N_{\tau}\left(E^{\prime \prime}\right)=\emptyset$ in $\Pi(\tau)$. This contradicts the fact that $i_{E} \circ \alpha_{K, 1}^{\prime \prime} \sim i_{E^{\prime \prime}} \circ \gamma_{K, 1}^{\prime \prime}$, so that we conclude that $E^{\prime \prime}=E^{\prime}$ and thus that $E^{\prime}$ is minimal as required.

The following theorem is the culmination of special cases proved earlier as Theorems $2.7,2.9$, and 3.4 .

Theorem 4.2. Let $G$ be a finite group scheme over $k$ and let

$$
\alpha_{K}: K[t] / t^{p} \rightarrow K G, \quad \beta_{L}: L[t] / t^{p} \rightarrow L G
$$

be equivalent generic $\pi$-points of $G$. Then for any finite dimensional $k G$-module $M, \alpha_{K}^{*}\left(M_{K}\right)$ has the same Jordan type as $\beta_{L}^{*}\left(M_{L}\right)$.

Proof. As seen in $[12,4.2]$, we may find a finite field extension $F / k$ such that $G_{F}$ satisfies the hypotheses of Proposition 4.1 that $G_{F}$ splits as a semi-direct product $G_{F}^{0} \rtimes \tau$, with $\tau=\pi_{0}\left(G_{F}\right)$ constant and $G_{F}^{0}$ geometrically connected. Thus, appealing to Lemma 2.8, we may assume that $G$ itself satisfies these conditions. 
Let $\left[\alpha_{K}\right] \in \Pi(G)$ be a generic point and choose some representative

$$
\alpha_{K}=i_{C} \circ \tilde{\alpha}_{K}: K[t] / t^{p} \stackrel{\tilde{\alpha}_{K}}{\longrightarrow} K C_{K} \stackrel{i_{C}}{\longrightarrow} K G
$$

with $C_{K} \subset G_{K}$ a unipotent abelian subgroup scheme. Let $E \subset \pi_{0}\left(C_{K}\right)$ be the maximal elementary abelian $p$-subgroup and observe that $\Pi\left(C_{K}^{0} \times E\right) \rightarrow \Pi\left(C_{K}\right)$ is a homeomorphism. Thus, we may choose $\tilde{\alpha}_{K}^{\prime}: K[t] / t^{p} \longrightarrow K\left(C_{K}^{0} \times E\right)$ such that when composed with the embedding $K\left(C_{K}^{0} \times E\right) \hookrightarrow K C_{K}$ this map becomes equivalent to $\tilde{\alpha}_{K}$ as a $\pi$-point of $C_{K}$. Let $\alpha_{K}^{\prime}=i \circ \tilde{\alpha}_{K}^{\prime}: K[t] / t^{p} \longrightarrow K\left(\left(G^{0}\right)^{E} \times E\right)$ where $i: K\left(C_{K}^{0} \times E\right) \hookrightarrow K\left(\left(G^{0}\right)^{E} \times E\right)$ is the embedding. By construction, the composition of $\alpha_{K}^{\prime}$ with the embedding $K\left(\left(G^{0}\right)^{E} \times E\right) \hookrightarrow K G$ is equivalent to $\alpha_{K}$ as a $\pi$-point of $G$. In view of (4.0.1), the fact that $\alpha_{K}$ is generic implies that $\alpha_{K}^{\prime}$ is a generic $\pi$-point of $\left(G^{0}\right)^{E} \times E$. By Proposition 4.1, we can find

$$
\alpha_{K}^{\prime \prime}: K[t] / t^{p} \longrightarrow K\left(\left(G^{0}\right)^{E} \times E^{\prime}\right)
$$

where $E^{\prime}$ is a subgroup of $E$ and such that $\left[\alpha_{K}^{\prime \prime}\right] \in \Pi_{0}\left(\left(G^{0}\right)^{E^{\prime}} \times E^{\prime}\right)$ and $\alpha_{K}^{\prime \prime} \sim \alpha_{K}^{\prime}$ as $\pi$-points of $\left(G^{0}\right)^{E} \times E$. The latter implies that $\alpha_{K}^{\prime \prime}$ is generic for $\left(G^{0}\right)^{E} \times E$. Since $k\left(\left(G^{0}\right)^{E} \times E\right) \simeq k\left(\left(G^{0}\right)^{E} \times \mathbb{G}_{a(1)}^{\times r}\right)$ where $r$ is the rank of $E$, we may apply Theorem 3.4 to $\left(G^{0}\right)^{E} \times E$. Thus,

$$
\alpha_{K}^{*}\left(M_{K}\right) \simeq \alpha_{K}^{\prime \prime *}\left(i^{\prime *} M_{K}\right)
$$

where $i^{\prime}:\left(G^{0}\right)^{E} \times E^{\prime} \hookrightarrow G$. Repeating this argument for $\beta_{L}$, we conclude that

$$
\beta_{L}^{*}\left(M_{L}\right) \simeq \beta_{L}^{\prime \prime *}\left(j^{\prime *} M_{L}\right)
$$

where $\beta_{L}^{\prime \prime}: L[t] / t^{p} \longrightarrow L\left(\left(G^{0}\right)^{F} \times F^{\prime}\right)$ is a generic $\pi$-point of $\left(G^{0}\right)^{F} \times F^{\prime}$ with $F^{\prime} \subset F$ elementary abelian subgroups of $\tau,\left[\beta_{L}^{\prime \prime}\right] \in \Pi_{0}\left(\left(G^{0}\right)^{F^{\prime}} \times F^{\prime}\right)$, and $j^{\prime} \circ \beta_{L}^{\prime \prime} \sim \beta_{L}$. Here, $j^{\prime}:\left(G^{0}\right)^{F} \times F^{\prime} \hookrightarrow G$.

We therefore obtain $\left[\alpha_{K}^{\prime \prime}\right] \in \Pi_{0}\left(\left(G^{0}\right)^{E^{\prime}} \times E^{\prime}\right),\left[\beta_{L}^{\prime \prime}\right] \in \Pi_{0}\left(\left(G^{0}\right)^{F^{\prime}} \times F^{\prime}\right)$ such that $\alpha_{K}^{\prime \prime}, \beta_{L}^{\prime \prime}$ become equivalent (generic) $\pi$-points of $G$ after composing with the embeddings of the corresponding subgroup schemes into $G$. This implies that $E^{\prime}$ and $F^{\prime}$ are conjugate by an element of $\tau$; otherwise

$$
\Pi_{0}\left(\left(G^{0}\right)^{E^{\prime}} \times E^{\prime}\right) / N_{\tau}\left(E^{\prime}\right) \cap \Pi_{0}\left(\left(G^{0}\right)^{F^{\prime}} \times F^{\prime}\right) / N_{\tau}\left(F^{\prime}\right)
$$

would be empty, which would contradict the Quillen decomposition (4.0.1). Thus, after replacing $\beta_{L}^{\prime \prime}$ by a conjugate, which does not affect the Jordan type, we may assume that $\alpha_{K}^{\prime \prime}, \beta_{L}^{\prime \prime}$ are equivalent generic $\pi$-points of $\left(G^{0}\right)^{E^{\prime}} \times E^{\prime}$. Applying Theorem 3.4 once again (using the isomorphism $k\left(\left(G^{0}\right)^{E^{\prime}} \times E^{\prime}\right) \simeq k\left(\left(G^{0}\right)^{E^{\prime}} \times \mathbb{G}_{a(1)}^{\times r^{\prime}}\right)$, where $r^{\prime}=\operatorname{rank} E^{\prime}$, we conclude that the Jordan type of $\alpha_{K}^{\prime \prime *}\left(i^{\prime *} M_{K}\right)$ equals that of $\beta_{L}^{\prime \prime *}\left(j^{\prime *} M_{L}\right)$, and hence the Jordan type of $\alpha_{K}^{*}\left(M_{K}\right)$ equals that of $\beta_{L}^{*}\left(M_{L}\right)$.

We emphasize the conclusion of Theorem 4.2 with the following definition. As we shall see in examples given at the end of this section, a given $k G$-module $M$ can have different Jordan types at different (equivalence classes of) generic $\pi$-points; moreover, at some generic points, the Jordan type need not be maximal.

Definition 4.3. Let $G$ be a finite group scheme and $M$ be a $k G$-module. A generic Jordan type of $M$ is the Jordan type of $\alpha_{K}^{*}\left(M_{K}\right)$ for some generic $\pi$-point $\alpha_{K}$ of 
$G$. We denote the isomorphism class of $\alpha^{*}\left(M_{K}\right)$ (as a $K[t] / t^{p}$-module) by

$$
\left[\alpha_{K}\right]^{*}\left(M_{K}\right) \text {. }
$$

For the purposes of the following proposition, we utilize the coproduct on the algebra $k[t] / t^{p}$ given by the formula $\nabla(t)=t \otimes 1+1 \otimes t$. We point out here that this is a temporary convention: as it will be shown in Corollary 4.5 and more generally in Remark 4.6, the Jordan type of a tensor product of finite dimensional $k[t] / t^{p}$-modules does not depend on the Hopf algebra structure given to $k[t] / t^{p}$.

Since a $\pi$-point is not necessarily a Hopf algebra map and not even always equivalent to one, the following proposition is somewhat striking. The conclusion of this proposition does not always hold for non-generic $\pi$-points.

Proposition 4.4. Let $C$ be a unipotent abelian finite group scheme over $k$ and let $\alpha_{K}: K[t] / t^{p} \rightarrow K C$ be a generic $\pi$-point of $C$. Then for any pair of finite dimensional $k C$-modules $M, N$,

$$
\alpha_{K}^{*}\left(M_{K} \otimes_{K} N_{K}\right) \simeq \alpha_{K}^{*}\left(M_{K}\right) \otimes_{K} \alpha_{K}^{*}\left(N_{K}\right)
$$

Proof. Because we may base change to the perfect closure of $k$ without changing the conclusion, we may assume that $k$ is perfect. Thus, we may assume that $k C \simeq k\left[t_{1}, \ldots, t_{r}\right] /\left(t_{1}^{p^{e_{1}}}, \ldots, t_{r}^{p^{e_{r}}}\right)($ see $[21,14.4])$. Set $T_{i}=t_{i}^{p^{e_{i}-1}}$. Then any $\pi$-point $\beta: k[t] / t^{p} \rightarrow k C$ sends $t$ to a polynomial in $\left\{t_{i}\right\}$ whose $p$-th power is trivial and thus each of whose monomials is divisible by some $T_{i}$; the flatness of $\beta$ is equivalent to the conditon that some monomial constituting $\beta(t)$ is a non-zero multiple of some $T_{i}$.

Let $\rho_{M}: k C \rightarrow \operatorname{End}(M)$ be the map defined by the representation $M$ of $C$, and similarly define $\rho_{N}$ and $\rho_{M \otimes N}$. Since $\alpha_{K}$ is generic, Theorem 2.9 implies that $\rho_{M}\left(\alpha_{K}(t)\right)$ is maximal among the images under $\rho_{M}$ of all $p$-nilpotent elements of the commutative algebra $k C$, and similarly for $N$. Proposition 1.3 implies the equivalences

$$
\rho_{M}\left(\alpha_{K}(t)\right) \sim \rho_{M}\left(\alpha_{K}(t)\right)+s_{1} \rho_{M}\left(T_{1}\right)+\cdots+s_{r} \rho_{M}\left(T_{r}\right)
$$

$$
\rho_{N}\left(\alpha_{K}(t)\right) \sim \rho_{N}\left(\alpha_{K}(t)\right)+s_{r+1} \rho_{N}\left(T_{1}\right)+\cdots+s_{2 r} \rho_{N}\left(T_{r}\right)
$$

where $s_{i}$ are indeterminants. Therefore,

$$
s_{r} \rho_{M}\left(T_{r}\right) \otimes 1+1 \otimes \rho_{N}\left(\alpha_{K}(t)\right)+1 \otimes s_{r+1} \rho_{N}\left(T_{1}\right)+\cdots+1 \otimes s_{2 r} \rho_{N}\left(T_{r}\right)
$$

Indeed, if $A$ is the matrix which makes the endomorphisms of 4.4 .1 similar, and $B$ is such a matrix for 4.4.2, then $A \otimes B$ makes the endomorphisms in 4.4.3 similar. Proposition 1.3 implies that $\rho_{M}\left(\alpha_{K}(t)\right) \otimes 1+1 \otimes \rho_{N}\left(\alpha_{K}(t)\right)$ is maximal with respect to $\left\{\rho_{M}\left(T_{i}\right) \otimes 1,1 \otimes \rho_{N}\left(T_{j}\right) ; 1 \leq i, j \leq r\right\}$. Thus, Theorem 1.12 implies that $\rho_{M}\left(\alpha_{K}(t)\right) \otimes 1+1 \otimes \rho_{N}\left(\alpha_{K}(t)\right)$ is maximal with respect to

(4.4.4) $\left\{\rho_{M}(p(\underline{t})) \otimes 1,1 \otimes \rho_{N}(q(\underline{t})) ; \quad\right.$ each monomial of $p, q$ divisible by some $\left.T_{i}\right\}$.

Let $\nabla: k C \rightarrow k C \otimes k C$ be the coproduct on $k C$, and let $I$ be the augmentation ideal of $k C$. Since $\nabla\left(\alpha_{K}(t)\right)-\alpha_{K}(t) \otimes 1-1 \otimes \alpha_{K}(t) \in I \otimes I$ (see [15, I.2.4]) and is $p$-nilpotent, this difference is a sum of terms each of which is either a product of the form $(p(\underline{t}) \otimes 1) \cdot(1 \otimes f(\underline{t}))$ with each monomial of $p(\underline{t})$ divisible by some $T_{i}$ and with $f$ having no constant term or a product of the form $(g(\underline{t}) \otimes 1) \cdot(1 \otimes q(\underline{t}))$ 
with $g$ having no constant term and each monomial of $q(\underline{t})$ divisible by some $T_{i}$. Theorem 1.12 and the maximality of $\rho_{M}\left(\alpha_{K}(t)\right) \otimes 1+1 \otimes \rho_{N}\left(\alpha_{K}(t)\right)$ with respect to (4.4.4) imply

$$
\rho_{M}\left(\alpha_{K}(t)\right) \otimes 1+1 \otimes \rho_{N}\left(\alpha_{K}(t)\right) \sim\left(\left(\rho_{M} \otimes \rho_{N}\right) \circ \nabla\right)\left(\alpha_{K}(t)\right)=\rho_{M \otimes N}\left(\alpha_{K}(t)\right) .
$$

Thus, $\alpha_{K}^{*}(M) \otimes_{K} \alpha_{K}^{*}(N)$ and $\alpha_{K}^{*}\left(M_{K} \otimes_{K} N_{K}\right)$ have the same Jordan type.

The following is an interesting, though very special case of Proposition 4.4 in which $\alpha_{K}: K[t] / t^{p} \rightarrow K C$ is the identity map id $: \mathrm{k}[\mathrm{t}] / \mathrm{t}^{\mathrm{p}} \rightarrow \mathrm{k}[\mathrm{t}] / \mathrm{t}^{\mathrm{p}}$.

Corollary 4.5. Let $C$ be a unipotent abelian finite group scheme with $k C=k[t] / t^{p}$ and let $M, N$ be finite dimensional $k C$-modules. Then the tensor product of $M, N$ as $k C$-modules is isomorphic as a $k[t] / t^{p}$-module with the tensor product $M \otimes N$ using the coproduct $\nabla(t)=t \otimes 1+1 \otimes t$.

Remark 4.6. The proof of Proposition 4.4 does not use the fact that $k C$ is the Hopf algebra of a group scheme: namely, we do not need cocommutativity of the coproduct. The only fact about the coproduct which is needed is that $\nabla\left(\alpha_{K}(t)\right)-$ $\alpha_{K}(t) \otimes 1-1 \otimes \alpha_{K}(t) \in I \otimes I$, a property which holds for the coproduct of any Hopf algebra (see, for example, ex.3 on p.19, [21]). With this in mind, we can strengthen the statement of the Corollary 4.5 as follows: Let $M, N$ be finite dimensional $k[t] / t^{p}$-modules, let $M \otimes N$ denote the $k[t] / t^{p}$-module determined by the coproduct $\nabla(t)=t \otimes 1+1 \otimes t$. Then for any other coproduct $\nabla^{\prime}: k[t] / t^{p} \rightarrow k[t] / t^{p} \otimes k[t] / t^{p}$ associated to a Hopf algebra structure on $k[t] / t^{p}$, the resulting $k[t] / t^{p}$-module is isomorphic to $M \otimes N$.

Proposition 4.4 provides the key verification which enables us to prove the following pleasing properties of generic Jordan type. Recall that the Heller shift of a $k G$-module $M$, denoted $\Omega M$, is defined to be the kernel of the surjection $P(M) \longrightarrow M$, where $P(M)$ is the minimal projective cover of $M$.

Proposition 4.7. Let $G$ be a finite group scheme over a field $k$ and let $\left[\alpha_{K}\right] \in \Pi(G)$ be a generic point for some $K / k$. Let $M, N$ be finite dimensional $k G$-modules.

- $\left[\alpha_{K}\right]^{*}\left(M_{K} \oplus N_{K}\right) \simeq\left[\alpha_{K}\right]^{*}\left(M_{K}\right) \oplus\left[\alpha_{K}\right]^{*}\left(N_{K}\right)$.

- $\left[\alpha_{K}\right]^{*}\left(M_{K} \otimes N_{K}\right) \simeq\left[\alpha_{K}\right]^{*}\left(M_{K}\right) \otimes\left[\alpha_{K}\right]^{*}\left(N_{K}\right)$.

- $\left[\alpha_{K}\right]^{*}\left(\Omega\left(M_{K}\right)\right)=\Omega\left(\left[\alpha_{K}\right]^{*}\left(M_{K}\right)\right)$ in the stable module category of finite dimensional $K[t] / t^{p}$-modules, $\operatorname{stmod}\left(K[t] / t^{p}\right)$.

Thus, $\left[\alpha_{K}\right]^{*}:(k G-\bmod ) \rightarrow\left(K[t] / t^{p}-\bmod \right)$ induces a functor on tensor triangulated categories

$$
\left[\alpha_{K}\right]^{*}:(\operatorname{stmod}(k G)) \rightarrow\left(\operatorname{stmod}\left(K[t] / t^{p}\right)\right) .
$$

Proof. The first property follows from the observation that $\alpha_{K}^{*}$ commutes with direct sums.

Let $\alpha_{K}: K[t] / t^{p} \rightarrow K G$ be a generic $\pi$-point and let $C_{K} \subset G_{K}$ be a unipotent abelian subgroup scheme through which $\alpha_{K}$ factors. Write $\alpha_{K}=i \circ \alpha_{K}^{\prime}: K[t] / t^{p} \rightarrow$ $K C_{K} \rightarrow K G$. If we replace $\alpha_{K}^{\prime}$ by a generic $\pi$-point $\alpha_{L}^{\prime \prime}: L[t] / t^{p} \rightarrow L C_{L}$ of $C_{K}$, the new composition $i \circ \alpha_{L}^{\prime \prime}$ represents the same generic point of $\Pi(G)$. Thus, $\left[\alpha_{K}\right]^{*}=\left[i \circ \alpha_{L}^{\prime \prime}\right]^{*}$. The second property now follows from the observation that $i$ is a Hopf algebra map and that Proposition 4.4 applies to $\alpha_{L}^{\prime \prime}$.

The third property follows from the exactness of $\alpha_{K}^{*}$ and the fact that $\alpha_{K}^{*}$ sends projectives to projectives. 
For an arbitrary finite group scheme $G$ with $\Pi(G)$ reducible, the following construction produces a finite dimensional $k G$-module $M$ which has at least two distinct generic Jordan types. We implicitly use Proposition 4.7 in the justification of this example.

Example 4.8. Let $G$ be any finite group scheme with $\Pi(G)$ reducible; for example, $G$ any finite group with at least two distinct conjugacy classes of maximal elementary abelian $p$-subgroups. Write $\Pi(G)=X \cup Y$ with $X, Y$ proper closed subsets and choose generic points $\left[\alpha_{K}\right] \in X,\left[\beta_{L}\right] \in Y$. Extending scalars, if necessary, we can assume $L=K$. Choose $\zeta \in \mathrm{H}^{2 i}(G, k), \xi \in \mathrm{H}^{2 j}(G, k)$ such that

$$
\alpha_{K}^{*}\left(\zeta_{K}\right)=0 \neq \beta_{K}^{*}\left(\zeta_{K}\right) \text { and } \alpha_{K}^{*}\left(\xi_{K}\right) \neq 0=\beta_{K}^{*}\left(\xi_{K}\right)
$$

(Here, $\alpha_{K}^{*}$ is the map in cohomology $\mathrm{H}^{*}(G, K) \rightarrow \mathrm{H}^{*}\left(K[t] / t^{p}, K\right)$ induced by $\alpha_{K}$. ) Let $L_{\zeta}, L_{\xi}$ be the "Carlson modules" associated to $\zeta, \xi$ (see [2, II.5.9]). By definition of $L_{\zeta}$, we have a short exact sequence

$$
0 \longrightarrow L_{\zeta} \longrightarrow \Omega^{2 i} k \stackrel{\tilde{\zeta}}{\longrightarrow} k \longrightarrow 0
$$

where the map $\tilde{\zeta}: \Omega^{2 i} k \rightarrow k$ is given by $\zeta$ via the isomorphism $\underline{\operatorname{Hom}}\left(\Omega^{2 i} k, k\right)=$ $\mathrm{H}^{2 i}(G, k)$. Extending scalars to $K$ and then pulling back the short exact sequence via the map $\alpha_{K}$, we get a short exact sequence of $K[t] / t^{p}$ - modules

$$
0 \longrightarrow \alpha_{K}^{*}\left(L_{\zeta, K}\right) \longrightarrow \Omega^{2 i} K \stackrel{\left(\tilde{\zeta}_{K}\right) \downarrow_{\alpha_{K}}}{\longrightarrow} K \longrightarrow 0
$$

Since $\alpha_{K}^{*}\left(\zeta_{K}\right)=0$, the map $\left(\tilde{\zeta}_{K}\right) \downarrow_{\alpha_{K}}: \Omega^{2 i} K \longrightarrow K$ factors through a projective $K[t] / t^{p}$ - module. Since $\Omega^{2 i} K=K \oplus \operatorname{proj}$ as $K[t] / t^{p}$ - modules, we conclude that the kernel $\alpha_{K}^{*}\left(L_{\zeta, K}\right)=K \oplus \Omega K \oplus$ proj . In other words, the Jordan type of $\alpha_{K}^{*}\left(L_{\zeta, K}\right)$ is $1[1]+1[p-1]+m[p]$, for some $m \geq 0$. Here, we denote by $[i]$ an indecomposable $K[t] / t^{p}$ - module of dimension $i$. Arguing similarly, we get that $\beta_{K}^{*}\left(L_{\zeta, K}\right)$ and $\alpha_{K}^{*}\left(L_{\xi, K}\right)$ are projective whereas $\beta_{K}^{*}\left(L_{\xi, K}\right)$ has Jordan form $1[1]+1[p-1]+n[p]$, for some $n \geq 0$.

Let $M=L_{\zeta} \oplus L_{\xi}^{\oplus 2}$. Then the generic Jordan types $\left[\alpha_{K}\right]^{*}\left(M_{K}\right),\left[\beta_{K}\right]^{*}\left(M_{K}\right)$ are different. Indeed, it is enough to show that they stably different, i.e. different ignoring projective summands. The stable Jordan type of $\left[\alpha_{K}\right]^{*}\left(M_{K}\right)$ equals that of $\left[\alpha_{K}\right]^{*}\left(L_{\zeta, K}\right)$ which is $1[1]+1[p-1]$, whereas the stable Jordan type of $\left[\beta_{K}\right]^{*}\left(M_{K}\right)$ equals that of $\left[\beta_{K}\right]^{*}\left(L_{\xi, K}^{\oplus 2}\right)$ which is $2[1]+2[p-1]$. In particular, $M_{K}$ has the absolute Jordan type $\left[\alpha_{K}\right]^{*}\left(M_{K}\right)$.

The following explicit example reflects the same behavior of generic Jordan types as the examples constructed in Example 4.8.

Example 4.9. Let $G=G L\left(3, \mathbb{F}_{p}\right)$ with $p>2$. The irreducible components of $\Pi(G)$ are indexed by the conjugacy classes of maximal elementary $p$-subgroups of $G$ which are represented by subgroups of the unipotent subgroup $U\left(3, \mathbb{F}_{p}\right)$ of strictly upper triangular matrices. There are 3 conjugacy classes, represented by the following subgroups: 


$$
\left\{\left(\begin{array}{ccc}
1 & a & b \\
0 & 1 & a \\
0 & 0 & 1
\end{array}\right) a, b \in \mathbb{F}_{p}\right\}\left\{\left(\begin{array}{ccc}
1 & a & b \\
0 & 1 & 0 \\
0 & 0 & 1
\end{array}\right) a, b \in \mathbb{F}_{p}\right\}\left\{\left(\begin{array}{ccc}
1 & 0 & b \\
0 & 1 & a \\
0 & 0 & 1
\end{array}\right) a, b \in \mathbb{F}_{p}\right\} .
$$

Let $M$ be the standard 3-dimensional (rational) representation of $G$. Then the generic Jordan type of $M$ indexed by the first of these maximal elementary abelian subgroups of $G$ is a single block of size 3, whereas the generic Jordan types indexed by each of the other conjugacy classes of maximal elementary abelian subgroups are strictly smaller.

We now present the extension of Theorem 3.5 to arbitrary finite group schemes.

Theorem 4.10. Let $G$ be a finite group scheme over $k$ and $M$ a finite dimensional $k G$-module. Let $\alpha_{K}: K[t] / t^{p} \rightarrow K G$ be a $\pi$-point of $G$ which has maximal Jordan type on $M$. Then for any $\pi$-point $\beta_{L}: L[t] / t^{p} \rightarrow L G$ which specializes to $\alpha_{K}$, the Jordan type of $\alpha_{K}^{*}\left(M_{K}\right)$ equals the Jordan type of $\beta_{L}^{*}\left(M_{L}\right)$; in particular, if $\alpha_{K} \sim \beta_{L}$, then the Jordan type of $\alpha_{K}^{*}\left(M_{K}\right)$ equals the Jordan type of $\beta_{L}^{*}\left(M_{L}\right)$.

Proof. We first consider the special case in which $\alpha_{K} \sim \beta_{L}$, and verify that $\beta_{L}^{*}\left(M_{L}\right)$ has the same (maximal for $M$ ) Jordan type as $\alpha_{K}^{*}\left(M_{K}\right)$. We proceed as in the proof of Theorem 4.2. Write

$$
\alpha_{K}=i_{C} \circ \alpha_{K}^{\prime}: K[t] / t^{p} \stackrel{\alpha_{K}^{\prime}}{\longrightarrow} K C_{K} \stackrel{i_{C}}{\longrightarrow} K G
$$

with $C_{K} \subset G_{K}$ a unipotent abelian subgroup scheme. By [10, 4.2], we may find some subgroup scheme $j: C_{K}^{0} \times E \subset C_{K}$ for some elementary abelian $p$-subgroup $E \subset \pi_{0}\left(C_{K}\right)$ and a $\pi$-point $\alpha_{\Omega}^{\prime \prime}: \Omega[t] / t^{p} \rightarrow \Omega\left(C_{K}^{0} \times E\right)$ for some field extension $\Omega / K$ such that

$$
j \circ \alpha_{\Omega}^{\prime \prime}: \Omega[t] / t^{p} \stackrel{\alpha_{\Omega}^{\prime \prime}}{\longrightarrow} \Omega\left(C_{K}^{0} \times E\right) \stackrel{j}{\longrightarrow} \Omega C_{K}
$$

is equivalent to $\alpha_{K}^{\prime}$ as a $\pi$-point of $C_{K}$, extending scalars if necessary. Since $\alpha_{K}$ has maximal Jordan type on $M$, the $\pi$-point $\alpha_{K}^{\prime}$ of $C_{K}$ has maximal Jordan type on the $C_{K}$-module $i_{C}^{*}\left(M_{K}\right)$ (see Remark 2.6). Hence, Theorem 2.9 implies that $\left(j \circ \alpha_{\Omega}^{\prime \prime}\right)^{*}\left(i_{C}^{*}\left(M_{\Omega}\right)\right)$ has the same (maximal for $\left.i_{C}^{*}\left(M_{\Omega}\right)\right)$ Jordan type as $\alpha_{K}^{\prime *}\left(i_{C}^{*}\left(M_{K}\right)\right)$, and, hence, as $\alpha_{K}^{*}\left(M_{K}\right)$. Extending scalars to have $K=\Omega$, we may now replace $\alpha_{K}$ by the equivalent $\pi$-point $\left(i_{C} \circ j\right) \circ \alpha_{K}^{\prime \prime}$ which has the same maximal Jordan type on $M$ as $\alpha_{K}$. Hence, we may assume that $\alpha_{K}$ factors as

$$
\alpha_{K}=i \circ \alpha_{K}^{\prime}: K[t] / t^{p} \stackrel{\alpha_{K}^{\prime}}{\longrightarrow} K\left(C_{K}^{0} \times E\right) \stackrel{i}{\longrightarrow} K G .
$$

Decompose $i: K\left(C_{K}^{0} \times E\right) \longrightarrow K G$ as

$$
i=i_{E} \circ i_{C, E}: K\left(C_{K}^{0} \times E\right) \stackrel{i_{C, E}}{\longrightarrow} K\left(\left(G^{0}\right)^{E} \times E\right) \stackrel{i_{E}}{\longrightarrow} K G,
$$

and let $\widetilde{\alpha}_{K}$ be the restriction of $\alpha_{K}$ to the subgroup scheme $\left(G^{0}\right)^{E} \times E$ of $G$ :

$$
\widetilde{\alpha}_{K}=i_{C, E} \circ \alpha_{K}^{\prime}: K[t] / t^{p} \stackrel{\alpha_{K}^{\prime}}{\longrightarrow} K\left(C_{K}^{0} \times E\right) \stackrel{i_{C, E}}{\longrightarrow} K\left(\left(G^{0}\right)^{E} \times E\right) .
$$

By Proposition 4.1, we may find $\widetilde{\alpha}_{K}^{\prime}: K[t] / t^{p} \longrightarrow K\left(\left(G^{0}\right)^{E} \times E^{\prime}\right)$ equivalent to $\widetilde{\alpha}_{K}$ as a $\pi$-point of $\left(G^{0}\right)^{E} \times E$ with the additional property that $\left[\widetilde{\alpha}_{K}^{\prime}\right] \in \Pi_{0}\left(\left(G^{0}\right)^{E^{\prime}} \times\right.$ 
$\left.E^{\prime}\right)$ for some $E^{\prime} \subset E$. Since $k\left(\left(G^{0}\right)^{E} \times E\right) \simeq k\left(\left(G^{0}\right)^{E} \times \mathbb{G}_{a(1)}^{\times r}\right)$ (where $r=$ $\operatorname{rank}(E))$, we may apply Theorem 3.5 to $\left(G^{0}\right)^{E} \times E$. We conclude that $\widetilde{\alpha}_{K}^{\prime}$ has the same (maximal) Jordan type on $i_{E, E^{\prime}}^{*}\left(M_{K}\right)$ as $\widetilde{\alpha}_{K}$ has on $i_{E}^{*}\left(M_{K}\right)$, where $i_{E, E^{\prime}}: K\left(\left(G^{0}\right)^{E} \times E^{\prime}\right) \hookrightarrow K G$. Since $\alpha_{K}=i_{E} \circ \widetilde{\alpha}_{K}$, we further conclude that the Jordan type of $\left(i_{E, E^{\prime}} \circ \widetilde{\alpha}_{K}^{\prime}\right)^{*}\left(M_{K}\right)$ is the same as of $\alpha_{K}^{*}\left(M_{K}\right)$. Hence, replacing $\alpha_{K}$ with the equivalent $\pi$-point $i_{E, E^{\prime}} \circ \widetilde{\alpha}_{K}^{\prime}$ which has the same (maximal) Jordan type on $M$, we may assume that $\alpha_{K}$ factors as

$$
\alpha_{K}=i_{E} \circ \widetilde{\alpha}_{K}: K[t] / t^{p} \stackrel{\widetilde{\alpha}_{K}}{\longrightarrow} K\left(\left(G^{0}\right)^{E} \times E\right) \stackrel{i_{E}}{\longrightarrow} K G
$$

with the additional property $\left[\widetilde{\alpha}_{K}\right] \in \Pi_{0}\left(\left(G^{0}\right)^{E} \times E\right)$ for some elementary abelian p-group $E \subset \pi_{0}(G)$.

Performing the same series of operations on $\beta_{L}$, we find a $\pi$-point $\beta_{L}^{\prime}$ equivalent to $\beta_{L}$ (possibly after some extensions of scalars) which factors as

$$
\beta_{L}^{\prime}=i_{F} \circ \widetilde{\beta}_{L}: L[t] / t^{p} \stackrel{\widetilde{\beta}_{L}}{\longrightarrow} L\left(\left(G^{0}\right)^{F} \times F\right) \stackrel{i_{F}}{\longrightarrow} L G
$$

with the additional property $\left[\widetilde{\beta}_{L}\right] \in \Pi_{0}\left(\left(G^{0}\right)^{F} \times F\right)$ for some elementary abelian p-group $F \subset \pi_{0}(G)$. (Observe that we do not claim yet that $\beta_{L}^{\prime}$ has the same Jordan type on $M$ as $\beta_{L}$.)

Using (4.0.1) as in the last paragraph of the proof of Theorem 4.2 we conclude that some $\pi_{0}(G)$-conjugate of $\widetilde{\beta}_{L}$ is equivalent to $\widetilde{\alpha}_{K}$ as a $\pi$-point of $\left(G^{0}\right)^{E} \times E$. Thus, Theorem 3.5 implies that $\widetilde{\beta}_{L}^{*}\left(i_{F}^{*}\left(M_{L}\right)\right.$ ) has the same (maximal for $M$ ) Jordan type as $\widetilde{\alpha}_{K}^{*}\left(i_{E}^{*}\left(M_{K}\right)\right) \simeq \alpha_{K}^{*}\left(M_{K}\right)$. Consequently, the preceding argument given for $\alpha_{K}$ now "run backwards" and applied to $\beta_{L}$ implies that $\beta_{L}$ has the same maximal Jordan type on $M$ as $\alpha_{K}$.

More generally, assume that $\beta_{L}$ specializes to $\alpha_{K}$ as a $\pi$-point of $G$ and construct $\beta_{L}^{\prime}=i_{F} \circ \widetilde{\beta}_{L}$ as in (4.10.1). Since the image of $\Pi\left(\left(G^{0}\right)^{F} \times F\right)$ in $\Pi(G)$ is closed, and since $\beta_{L}^{\prime} \sim \beta_{L}$ specializes to $\alpha_{K}$, we can find $\gamma_{K}: K[t] / t^{p} \rightarrow K\left(\left(G^{0}\right)^{F} \times F\right)$ (possibly after a scalar extension) such that $i_{F} \circ \widetilde{\beta}_{L}$ specializes to $i_{F} \circ \gamma_{K}$, and $i_{F} \circ \gamma_{K} \sim \alpha_{K}$ as $\pi$-points of $G$.

The Quillen decomposition (4.0.1) implies that $\widetilde{\beta}_{L}$ specializes to a $\pi_{0}(G)$-conjugate of $\gamma_{K}$ as a $\pi$-point of $\left(G^{0}\right)^{F} \times F$. Since $i_{F} \circ \gamma_{K} \sim \alpha_{K}$, the just proved special case (in which $\left.\beta_{L} \sim \alpha_{K}\right)$ implies that $\gamma_{K}^{*}\left(i_{F}^{*}\left(M_{K}\right)\right.$ ) has the same maximal Jordan type for $M$ as $\alpha_{K}^{*}\left(M_{K}\right)$. Hence, $\gamma_{K}$ has maximal Jordan type on $i_{F}^{*}(M)$ by Remark 2.6. Thereom 3.5 applied to $\left(G^{0}\right)^{F} \times F$ implies that $\left(\beta_{L}^{\prime}\right)^{*}\left(M_{L}\right) \simeq \widetilde{\beta}_{L}\left(i_{F}^{*}\left(M_{L}\right)\right)$ has the same (maximal for $i_{F}^{*}\left(M_{L}\right)$ ) Jordan type as $\gamma_{K}^{*}\left(i_{F}^{*}\left(M_{K}\right)\right)$ which, in turn, is the same as the Jordan type of $\alpha_{K}^{*}\left(M_{K}\right)$. Since $\beta_{L} \sim \beta_{L}^{\prime}$, we conclude by another application of the case of equivalent $\pi$-points that $\beta_{L}^{*}\left(M_{L}\right)$ has the same (maximal for $M)$ Jordan type as $\alpha_{K}^{*}\left(M_{K}\right)$.

The following corollary follows immediately from Theorem 4.10.

Corollary 4.11. Let $G$ be a finite group scheme and $M$ a finite dimensional $k G$ module. Then each maximal Jordan type of $M$ can be realized as the Jordan type of $\alpha_{K}^{*}\left(M_{K}\right)$ for some generic point $\left[\alpha_{K}\right] \in \Pi(G)$.

In particular, if $\Pi(G)$ is irreducible, then for any finite dimensional $k G$-module $M$ the Jordan type of $\alpha_{K}^{*}(M)$ is the absolute maximal type for $M$ whenever $\alpha_{K}$ represents the generic point of $\Pi(G)$. 
In order to consider examples obtained by induction from subgroup schemes, we shall employ the following well known property of support varieties. For lack of a good reference, we provide a simple proof of this result. Recall that if $H \subset G$ is a closed subgroup scheme and $N$ an $H$-module, then the induced module $\operatorname{Ind}_{H}^{G}(N)$ is the $G$-module with underlying vector space $(N \otimes k[G])^{H}$, with $G$ action given by the left regular action of $G$ of $k[G]$ and with $H$-invariants taken with respect to the given action on $N$ and the right regular action on $k[G]$.

Lemma 4.12. Let $G$ be a finite group scheme and $i: H \subset G$ a closed subgroup scheme. Let $N$ be a finite dimensional $k H$-module, and let $M=\operatorname{Ind}_{H}^{G}(N)$. Then the natural map $i: \Pi(H) \rightarrow \Pi(G)$ satisfies the property

$$
\Pi(G)_{M} \subset i\left(\Pi(H)_{N}\right) .
$$

Proof. Observe that the action of $\mathrm{H}^{\bullet}(G, k)$ on $\operatorname{Ext}_{G}^{*}(M, M)$ factors as the composition of the restriction $\mathrm{H}^{\bullet}(G, k) \rightarrow \mathrm{H}^{\bullet}(H, k)$ followed by the natural action of $\mathrm{H}^{\bullet}(H, k)$ on $\operatorname{Ext}_{H}^{*}\left(N, M \downarrow_{H}\right)=\operatorname{Ext}_{G}^{*}(M, M)$. Hence, a homogeneous prime ideal of $\mathrm{H}^{\bullet}(G, k)$ contains the annihilator of $\operatorname{Ext}_{G}^{*}(M, M)$ (and hence lies in Proj $|G|_{M}=$ $\left.\Pi(G)_{M}\right)$ if and only if it is the pre-image of a homogeneous prime ideal of $\mathrm{H}^{\bullet}(H, k)$ containing the annihilator of $\operatorname{Ext}_{H}^{*}\left(N, M \downarrow_{H}\right)$ and the latter are among the homogeneous prime ideals of $\mathrm{H}^{\bullet}(H, k)$ containing the annihilator of $\operatorname{Ext}_{H}^{*}(N, N)$ (i.e., an element of Proj $\left.|H|_{N}=\Pi(H)_{N}\right)$ since the action of $\mathrm{H}^{*}(H, k)$ on $\operatorname{Ext}_{H}^{*}\left(N, M \downarrow_{H}\right)$ factors through $\operatorname{Ext}_{H}^{*}(N, N)$.

To complement Corollary 4.11, we give an example of a finite dimensional $k G$ module for which there is no absolute maximal Jordan type.

Example 4.13. Let $G$ be a finite $p$-group which has two conjugacy classes of maximal elementary abelian subgroups, represented by $E$ and $E^{\prime}$ respectively. Furthermore, we require $E$ to be normal. Let $e=\# E, f=\frac{\# G}{\# E}$. Assume $p>3$.

For example, take $G$ to be the $p$-Sylow subgroup of the wreath product $\mathbb{Z} / p \mathbb{Z} \imath S_{p}$, so that $G$ is isomorphic to $(\mathbb{Z} / p \mathbb{Z})^{p} \rtimes \mathbb{Z} / p \mathbb{Z}$. Then $G$ has two conjugacy classes of maximal elementary abelian $p$-subgroups represented by $E=(\mathbb{Z} / p \mathbb{Z})^{\times p}$ (which is normal) and $F=(\mathbb{Z} / p \mathbb{Z} \times \mathbb{Z} / p \mathbb{Z} \times \cdots \times \mathbb{Z} / p \mathbb{Z})^{\mathbb{Z} / p \mathbb{Z}} \times \mathbb{Z} / p \mathbb{Z}$.

By Quillen stratification, $\Pi(G)=X \cup Y$ where $X=\Pi(E) / G, Y=\Pi(F) / N_{G}(F)$. Let $\left[\alpha_{K}\right] \in X,\left[\beta_{L}\right] \in Y$ be generic points. As usual, we may assume $L=K$ after scalar extension.

Let $n$ be a positive integer, let $M=\operatorname{Ind}_{E}^{G}\left(\Omega_{E} k\right)$, and let $N=L_{\xi}^{\oplus n}$ where $L_{\xi}$ is a Carlson module such that $\left[\beta_{K}\right] \in \Pi(G)_{L_{\xi}}$ but $\left[\alpha_{K}\right] \notin \Pi(G)_{L_{\xi}}$. This can be achieved by choosing $\xi \in \mathrm{H}^{2 N}(G, k)$ with the property that the restriction of $\xi$ to $\mathrm{H}^{2 N}(F, k)$ is 0 and to $\mathrm{H}^{2 N}(E, k)$ is non-zero.

Since $E$ is normal in $G$, the double coset formula implies that $M \downarrow_{E} \simeq\left(\Omega_{E} k\right)^{\oplus f}$. Since $\left[\alpha_{K}\right]$ is a generic $\pi$-point of $E$, a representative $\alpha_{K}$ can be chosen to factor through $k E$. Hence, the Jordan type of $\alpha_{K}^{*}\left(\Omega_{E} k\right)$ is

$$
1[p-1]+\frac{e-p}{p}[p]
$$

and the dimension is $e-1$. Hence, the Jordan type of $\alpha_{K}^{*}\left(M_{K}\right)$ is

$$
f[p-1]+f \cdot \frac{e-p}{p}[p] .
$$

Moreover, $\beta_{K}^{*}\left(M_{K}\right)$ is projective (of dimension is $(e-1) f$ ) by Lemma 4.12. 
Let $\ell=\frac{\operatorname{dim} L_{\xi}-p}{p}$. Arguing as in Example 4.8, we get that $\alpha_{K}^{*}\left(L_{\xi, K}\right)$ is projective (of dimension $p(\ell+1)$ ), and the Jordan type of $\beta_{K}^{*}\left(L_{\xi, K}\right)$ is $1[1]+1[p-1]+\ell[p]$. Hence, $\alpha_{K}^{*}\left(N_{K}\right)$ is projective of dimension $n(\ell+1) p$, and the Jordan type of $\beta_{K}^{*}\left(N_{K}\right)$ is

$$
n[1]+n[p-1]+n \ell[p] .
$$

We now compute the Jordan types of the module $M \oplus N$ at the generic points $\alpha_{K}$ and $\beta_{L}$. We have

$$
\begin{gathered}
\alpha_{K}^{*}\left((M \oplus N)_{K}\right) \simeq \alpha_{K}^{*}\left(M_{K}\right) \oplus \alpha_{K}^{*}\left(N_{K}\right) \simeq f[p-1]+f \cdot \frac{e-p}{p}[p]+n(\ell+1)[p] \simeq \\
f[p-1]+\left(\frac{e-p}{p} f+n(\ell+1)\right)[p]
\end{gathered}
$$

and

$$
\begin{gathered}
\beta_{K}^{*}\left((M \oplus N)_{K}\right) \simeq \beta_{K}^{*}\left(M_{K}\right) \oplus \beta_{K}^{*}\left(N_{K}\right) \simeq \frac{(e-1) f}{p}[p]+n[1]++n[p-1]+n \ell[p] \simeq \\
n[1]+n[p-1]+\left(\frac{(e-1) f}{p}+n \ell\right)[p] .
\end{gathered}
$$

Observe that for such two Jordan types to be incomparable it suffices for $\alpha_{K}^{*}((M \oplus$ $\left.N)_{K}\right)$ to have fewer blocks of size $[p]$ and fewer blocks altogether than $\beta_{K}^{*}((M \oplus$ $N)_{K}$ ). This amounts to two inequalities

$$
\frac{(e-p) f}{p}+n(\ell+1)<\frac{(e-1) f}{p}+n l
$$

and

$$
f+\frac{(e-p) f}{p}+n(\ell+1)<2 n+\frac{(e-1) f}{p}+n \ell .
$$

This reduces to

$$
\frac{f}{p}<n<(p-1) \frac{f}{p} .
$$

Since $p>3$ divides $f$, there exists $n$ satisfying the inequalities 4.13.1. For such $n$, the corresponding module $M \oplus N$ has incomparable generic Jordan types at the points $\alpha_{K}$ and $\beta_{K}$.

The conclusion of Theorem 4.10 is not valid for generic Jordan types. Namely, as the following example shows, one can find a generic $\pi$-point $\alpha_{K}$ of a finite group scheme $G$, a $\pi$-point $\beta$ which is a specialization of $\alpha_{K}$ and a $G$-module $M$ such that the Jordan type of $\beta^{*}(M)$ is strictly greater than that of $\alpha_{K}^{*}\left(M_{K}\right)$. According to Corollary 4.11 , in any such example $\Pi(G)$ must be reducible.

Example 4.14. We return to the example $G=(\mathbb{Z} / p \mathbb{Z})^{p} \rtimes \mathbb{Z} / p \mathbb{Z}$ of Example 4.13 and retain the same notation $\Pi(G)=X \cup Y, E, F, e, f$. Let $M=\operatorname{Ind}_{E}^{G} k$. Then the double coset formula implies that $M \downarrow_{E}$ is trivial, so that $\Pi(G)_{M}=X$ by Lemma 4.12. Let $\left\{g_{1}, \ldots, g_{p}\right\}$ be generators of $E, t_{i}=g_{i}-1$ and $K=k\left(s_{1}, \ldots, s_{p}\right)$. Let $\alpha_{K}: K[t] / t^{p} \rightarrow K E \rightarrow K G$ be a generic $\pi$-point of $G$ corresponding to the component $X$ defined by

$$
\alpha_{K}(t)=s_{1} t_{1}+\cdots+s_{p} t_{p} .
$$


The Jordan type of $\alpha_{K}^{*}\left(M_{K}\right)$ is trivial. Let $\beta$ be a $k$-rational $\pi$-point of $G$ representing the point on the intersection $X \cap Y$ given by

$$
\beta(t)=t_{1}+t_{2}+\cdots+t_{p}
$$

Since $E$ acts trivially on $M$, the Jordan type of $\beta^{*}(M)$ is trivial.

Let $\gamma: k[t] / t^{p} \rightarrow k F \rightarrow k G$ be a $k$-rational $\pi$-point of $G$ such that $[\gamma] \in Y,[\gamma] \notin$ $X$. Since $\Pi(G)_{M} \subset X$, we conclude that $\gamma^{*}(M)$ is projective. Since both $\gamma$ and $\beta$ factor through $k F, \beta(t)$ and $\gamma(t)$ commute. Define a new $\pi$-point $\tilde{\beta}$ by

$$
\tilde{\beta}(t)=\beta(t)+\gamma^{2}(t) .
$$

By Proposition 2.2, $[\beta]=[\tilde{\beta}]$ in $\Pi(G)$ and, hence, $\tilde{\beta}$ is a specialization of $\alpha_{K}$. On the other hand, since $\gamma^{*}(M)$ is projective and $p>2, \tilde{\beta}^{*}(M)$ is non-trivial. Thus, the Jordan type of $\tilde{\beta}^{*}(M)$ is greater than that of $\alpha_{K}^{*}\left(M_{K}\right)$.

As we see in the following example, the maximal Jordan type can sometimes be easily determined as a familiar invariant associated to a given $k G$-module $M$.

Example 4.15. Let $\mathcal{G}$ be a reductive algebraic group over $k$ with Lie algebra $g$. Let $G=\mathcal{G}_{(1)}$, so that $k G \simeq u(g)$, the restricted enveloping algebra of $g=\operatorname{Lie}(\mathcal{G})$. Then the maximal Jordan type of a finite dimensional $k G$-module $M$ is the Jordan type of any regular nilpotent element $X \in g$ acting upon $M$ provided that every nilpotent element of $g$ is $p$-nilpotent.

Consider the special case $G=S L_{n(1)}$ and let $M$ be the standard $n$-dimensional representation of $S L_{n}$ restricted to $G$. We make no assumption on the size of $p$ with respect to $n$ (i.e., we do not assume that every nilpotent element of $s l_{n}$ is $p$-nilpotent). Each $p$-nilpotent $X \in s l_{n}$ determines a 1-parameter subgroup $\mu_{X}: \mathbb{G}_{a(1)} \rightarrow G$. The Jordan type of $\left(\mu_{X}\right)^{*}(M)$ is merely the Jordan type of $X$ itself. The maximal Jordan type for $M$ is that given by the partition given by $\left[\frac{n}{p}\right]$ blocks of size $p$ and one block of size $n-p\left[\frac{n}{p}\right]$.

More generally, let $G=S L_{n(r)}, r \geq 1$, with $M$ once again the standard representation of $S L_{n}$ restricted to $G$. Since any $\pi$-point of $S L_{n(1)}$ determines a $\pi$ point of $S L_{n(r)}$ by composition with the closed (flat) embedding $S L_{n(1)} \subset S L_{n(r)}$, the maximal Jordan type for $M \downarrow_{S L_{n(1)}}$ is at most the maximal Jordan type for $M \downarrow_{S L_{n(r)}}$. On the other hand, the maximal Jordan type for $M \downarrow_{S L_{n(1)}}$ is maximal possible of any module of dimension $n$. Thus, the maximal Jordan type for $M$ as $S L_{n(r)}$-module remains the partition with $\left[\frac{n}{p}\right]$ blocks of size $p$ and one block of size $n-p\left[\frac{n}{p}\right]$.

The following most elementary example of an infinitesimal group scheme of height bigger than 1 gives a first indication of the behavior of generic Jordan type for infinitesimal group schemes.

Example 4.16. Let $G=\mathbb{G}_{a(r)}$ for some $r>s>1$, and let $u_{1}, \ldots, u_{r}$ be the standard algebraic generators of $k G$ as described in $\S 3$. Consider a finite dimensional $k \mathbb{G}_{(r-s)}$-module $M$ and the pull-back $p^{*} M=M^{(s)}$ via the projection $p: \mathbb{G}_{a(r)} \rightarrow \mathbb{G}_{a(r)} / \mathbb{G}_{a(s)} \simeq \mathbb{G}_{a(r-s)}$ sending $u_{i} \in k \mathbb{G}_{a(r)}$ to $u_{i-s} \in k \mathbb{G}_{a(r-s)}$ for $i>s$ and 0 otherwise. Observe that the action of $u_{i} \in k \mathbb{G}_{a(r)}$ on $M^{(s)}$ can be identified with the action of $u_{i-s} \in k \mathbb{G}_{a(r-s)}$ on $M$. We readily verify that the generic Jordan 
type of the $k \mathbb{G}_{(r-s)}$-module $M$ is equal to the generic Jordan type of the $k \mathbb{G}_{a(r)^{-}}$ module $M^{(s)}$. Namely, the generic type of $M$ is determined by the action of a generic linear combination of the generators $u_{i-s} \in k \mathbb{G}_{a(r-s)}$, and the generic type of $M^{(s)}$ is determined by the action of a generic linear combination of $u_{i} \in k \mathbb{G}_{a(r)}$. But since $u_{i} \in k \mathbb{G}_{a(r)}, i<s$ acts trivially on $M^{(s)}$ and $u_{i} \in k \mathbb{G}_{a(r)}, i \geq s$, acts as $u_{i-s} \in k \mathbb{G}_{a(r-s)}$ acts on $M$, these generic linear combinations clearly yield the same Jordan type.

\section{NON-MAXIMAL SUPPORT VARIETIES}

In this section we refine the construction of support varieties for modules over a finite group scheme. Cohomological support varieties were first introduced in the case of finite groups by J. Carlson [3], and subsequently extended by various authors, initially to restricted Lie algebras, then to infinitesimal group schemes, and finally to arbitrary finite group schemes. Carlson conjectured and G. Avrunin and L. Scott proved [1] that for elementary abelian $p$-groups the cohomological support variety of a module had a representation theoretic interpretation as the rank variety. This was subsequently generalized, with the ultimate formulation being the assertion of the existence of the naturally constructed homeomorphism (2.1.1) restricting to a homeomorphism of support varieties

$$
\Pi(G)_{M} \simeq \operatorname{Proj}|G|_{M}
$$

for any finite group scheme $G$ and any finite dimensional $k G$-module $M$ [12].

If the dimension of $M$ is not divisible by $p$, then the support variety $\Pi(G)_{M}$ is necessarily equal to $\Pi(G)$. In this section, we introduce a refinement $\Gamma(G)_{M} \subset \Pi(G)$ of $\Pi(G)_{M} \subset \Pi(G)$ which does give information about a $k G$-module $M$ even when the dimension of $M$ is not divisible by $p$. On the other hand, if the (absolute) maximal Jordan type of $M$ is projective, then the absolute maximal Jordan type for $M$ is the type of a projective $K[t] / t^{p}$-module so that the non-maximal support variety of $M$ equals the support variety of $M: \Gamma(G)_{M}=\Pi(G)_{M}$.

Definition 5.1. Let $G$ be a finite group scheme over $k$ and $M$ be a finite dimensional $k G$-module. We define

$$
\Gamma(G)_{M} \subset \Pi(G)
$$

to be the set of those equivalence classes $\left[\alpha_{K}\right] \in \Pi(G)$ such that $\alpha_{K}$ does not have the maximal Jordan type on $M$ for some choice $\alpha_{K}: K[t] / t^{p} \rightarrow K G$ of representative of $\left[\alpha_{K}\right]$.

Observe that Theorem 4.10 verifies that $\left[\alpha_{K}\right] \in \Gamma(G)_{M}$ if and only if for every choice of representative $\alpha_{K}$ of $\left[\alpha_{K}\right]$ the Jordan type of $\alpha_{K}^{*}(M)$ is not maximal for $M$.

Theorem 5.2. Let $G$ be a finite group scheme over $k$ and $M$ be a finite dimensional $k G$-module. Then $\Gamma(G)_{M}$ is a closed subspace of $\Pi(G)$, the "non-maximal support variety" of $M$.

Proof. We first consider the special case in which $G$ is infinitesimal of height $r$. Let $A$ denote the coordinate algebra $k\left[V_{r}(G)\right]$ and consider the universal 1-paramter subgroup (3.0.1),

$$
u: \mathbb{G}_{a(r), A} \longrightarrow G_{A},
$$




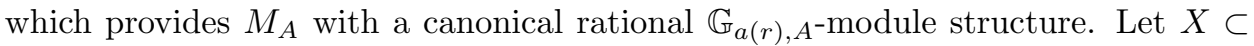
$V_{r}(G)$ be the subset of 1-parameter subgroups $\mu: \mathbb{G}_{a(r), K} \rightarrow G_{K}$ such that the $\pi$-point $\mu \circ \epsilon$ does not have maximal Jordan type on $M$. Then $X$ consists of those points $x \in V_{r}(G)$ for which the Jordan type of $\epsilon^{*}\left(u^{*}\left(M_{A}\right) \otimes_{A} k(x)\right)$ is not maximal for $M$.

Let $f_{A}: A[t] / t^{p} \longrightarrow \operatorname{End}_{A}(M)$ be the composition

$$
f_{A}: A[t] / t^{p} \stackrel{\epsilon}{\longrightarrow} A \mathbb{G}_{a(r)} \stackrel{u}{\longrightarrow} A G \stackrel{\rho_{M}}{\longrightarrow} \operatorname{End}_{A}(M) .
$$

Then $x \in X$ if and only if the Jordan type of $\left(f_{A} \otimes_{A} k(x)\right)(t) \in \operatorname{End}_{k(x)}(M \otimes k(x))$ is not maximal for $M$. This is described by a set of equations on the ranks of powers of $f_{A}(t)$. Thus, $X$ is the locus of points of $V_{r}(G)=\operatorname{Spec} A$ for which these equations admit a solution.

For a general finite group scheme $G$, we extend scalars to be able to assume that the Quillen decomposition (4.0.1) applies to $G$. Then $\Pi(G)$ is the finite union of the images of the closed maps $\Pi\left(\left(G^{0}\right)^{E} \times E\right) \rightarrow \Pi(G)$ as $E \subset \tau=\pi_{0}(G)$ varies over conjugacy classes of elementary abelian $p$-subgroups of $\tau$. Thus, for a given finite dimensional $k G$-module $M, \Gamma(G)_{M}$ consists of the union of the images of $\Pi\left(\left(G^{0}\right)^{E} \times E\right)$ indexed by those conjugacy classes such that the Jordan type of $M$ at the generic point of $\left(G^{0}\right)^{E} \times E$ is not maximal and the images of $\Gamma\left(\left(G^{0}\right)^{E} \times E\right)_{M} \subset$ $\Pi\left(\left(G^{0}\right)^{E} \times E\right)$ indexed by those conjugacy class such that the Jordan type of $M$ at the generic point of $\left(G^{0}\right)^{E} \times E$ is maximal. Consequently, it suffices to prove that each $\Gamma\left(\left(G^{0}\right)^{E} \times E\right)_{M} \subset \Pi\left(\left(G^{0}\right)^{E} \times E\right)$ is closed. Since $\left.k\left(\left(G^{0}\right)^{E} \times E\right)\right) \simeq$ $k\left(\left(G^{0}\right)^{E} \times \mathbb{G}_{a(1)}^{\times r}\right)$ where $r$ is the rank of $E$, this follows from the verification in the preceding paragraph for infinitesimal group schemes (applied to $\left(G^{0}\right)^{E} \times \mathbb{G}_{a(1)}^{\times r}$ ).

Proposition 5.3. Let $G$ be a finite group scheme over $k$ and $M, N$ be finite dimensional $k G$-modules, and $f: H \rightarrow G$ be a flat map of finite group schemes Then

(1) $\Gamma(G)_{M \oplus N} \subset \Gamma(G)_{M} \cup \Gamma(G)_{N}$. Moreover, if $\Pi(G)$ is irreducible, then equality holds.

(2) $\Gamma(H)_{f^{*} M} \subset\left(f_{*}\right)^{-1}\left(\Gamma(G)_{M}\right)$ where $f_{*}: \Pi(H) \rightarrow \Pi(G)$ is the map of schemes induced by $f$.

Proof. Let $\alpha_{K}: K[t] / t^{p} \rightarrow K G$ be a $\pi$-point with the property that $\alpha_{K}^{*}\left(M_{K}\right)$ has maximal Jordan type for $M$ and $\alpha_{K}^{*}\left(N_{K}\right)$ has maximal Jordan type for $N$, that is $\left[\alpha_{K}\right] \notin \Gamma(G)_{M} \cup \Gamma(G)_{N}$. Suppose, $\alpha_{K}^{*}\left(M_{K} \oplus N_{K}\right)$ does not have maximal Jordan type for $M_{K} \oplus N_{K}$. Then there exists a $\pi$-point $\beta_{L}$ such that $\alpha_{K}^{*}\left(M_{K} \oplus\right.$ $\left.N_{K}\right)<\beta_{L}^{*}\left(M_{L} \oplus N_{L}\right)$. This in turn implies that either $\alpha_{K}^{*}\left(M_{K}\right)<\beta_{L}^{*}\left(M_{L}\right)$ or $\alpha_{K}^{*}\left(N_{K}\right)<\beta_{L}^{*}\left(N_{L}\right)$ which contradicts maximality of Jordan types of $\alpha_{K}^{*}\left(M_{K}\right)$ and $\alpha_{K}^{*}\left(N_{K}\right)$. We conclude that the Jordan type of $\alpha_{K}^{*}\left(M_{K} \oplus N_{K}\right)$ is maximal for $M \oplus N$ which implies that $\left[\alpha_{K}\right] \notin \Gamma(G)_{M \oplus N}$.

Assume $\Pi(G)$ is irreducible with generic $\pi$-point $\beta_{L}$. To prove that some $\left[\alpha_{K}\right] \in$ $\Gamma(G)_{M} \cup \Gamma(G)_{N}$ is an element of $\Gamma(G)_{M \oplus N}$, it suffices to assume that $\alpha_{K}^{*}\left(M_{K}\right)$ is not maximal for $M$, that is $\alpha_{K}^{*}\left(M_{K}\right)<\beta_{L}^{*}\left(M_{L}\right)$. We have $\alpha_{K}^{*}\left(N_{K}\right) \leq \beta_{L}^{*}\left(N_{L}\right)$ since $\beta_{L}$ is generic. The maximality condition on ranks which defines the partial ordering (1.1.1) implies that $\alpha_{K}^{*}\left(M_{K} \oplus N_{K}\right)<\beta_{L}^{*}\left(M_{L} \oplus N_{L}\right)$.

To prove the second assertion, assume that the equivalence class of a $\pi$-point $\alpha_{K}$ of $H$ is not in $f_{*}^{-1}\left(\Gamma(G)_{M}\right)$. Equivalently, $f \circ \alpha_{K}$ has maximal Jordan type on $M$. 
This implies that the Jordan type of $\left(f \circ \alpha_{K}\right)^{*}\left(M_{K}\right) \simeq \alpha_{K}^{*}\left(f^{*} M\right)_{K}$ is maximal for $M$ among all $\pi$-points of $G$. In particular, it is maximal for $M$ among all $\pi$-points of the form $f \circ \beta_{L}$ where $\beta_{L}$ is a $\pi$-point of $H$. Hence, $\alpha_{K}$ has maximal Jordan type on the $H$-module $f^{*} M$. We conclude that $\left[\alpha_{K}\right] \notin \Gamma(H)_{f^{*} M}$, which implies the second assertion.

The following example demonstrates that the containment of statement (1) of Proposition 5.3 can not be sharpened to an equality.

Example 5.4. Let $G$ be as in Example 4.8, $M_{1}=L_{\zeta}, M_{2}=L_{\xi}^{\oplus 2}$. Then $\Gamma(G)_{M_{1}}=\Pi(G)_{M_{1}}, \Gamma(G)_{M_{2}}=\Pi(G)_{M_{2}}$, so that $\Gamma(G)_{M_{1}} \cup \Gamma(G)_{M_{2}}=\Pi(G)$, whereas $\Gamma(G)_{M_{1} \oplus M_{2}} \neq \Pi(G)$. Thus,

$$
\Gamma(G)_{M_{1} \oplus M_{2}} \neq \Gamma(G)_{M_{1}} \cup \Gamma(G)_{M_{2}} .
$$

On the other hand, if both $M$ and $N$ have maximal Jordan types which are projective, then

$$
\Gamma(G)_{M \oplus N}=\Pi(G)_{M \oplus N}=\Pi(G)_{M} \cup \Pi(G)_{N}=\Gamma(G)_{M} \cup \Gamma(G)_{N} .
$$

The following examples involve the consideration of the standard $n$-dimensional representation of $S L_{n}$. For these examples, determination of the non-maximal support variety is particularly easy, but the behavior of these varieties is illustrative.

Example 5.5. Let $G=S L_{n(1)}$ and let $M$ be the standard $n$-dimensional representation of $S L_{n}$ restricted to $G$. Recall that $\Pi\left(G_{(1)}\right)=\operatorname{Proj}\left(\mathcal{N}_{p}\right)$, the result of applying "proj" to the homogeneous closed subvariety $\mathcal{N}_{p} \subset s \ell_{n}$ of $p$-nilpotent matrices $([9],[20])$. By Example 4.15, the non-maximal support variety is homeomorphic to the subset of $\operatorname{Proj}\left(\mathcal{N}_{p}\right)$ consisting of equivalence classes of $p$-nilpotent matrices whose Jordan type is less than the type consisting of $\left[\frac{n}{p}\right]$ blocks of size $p$ and one block of size $n-p\left[\frac{n}{p}\right]$. This agrees with $\Pi(G)_{M} \subset \Pi(G)$ if and only if $n$ is divisible by $p$. For $p \geq n$, the non-maximal support variety is the complement inside the nullcone $\mathcal{N}$ (of all nilpotent matrices) of the open subset of regular nilpotent matrices.

Example 5.6. As in Example 4.9, let $G$ be $G L\left(3, \mathbb{F}_{p}\right)$ with $p>3$ and $M$ be the standard 3-dimensional $G L_{n}$-module $M$ restricted to $G L\left(n, \mathbb{F}_{p}\right)$. By the computation of Example 4.9, the non-maximal support variety is the union of two irreducible components.

\section{REFERENCES}

[1] G. Avrunin, L. Scott, Quillen stratification for modules, Invent. Math. 66 (1982) 277-286.

[2] D.J. Benson, Representations and cohomology, Volume I and II, Cambridge University Press, (1991).

[3] J. Carlson, The varieties and the cohomology ring of a module, J. Algebra 85 (1983) 104-143.

[4] J. Carlson, E. Friedlander, J. Pevtsova, Modules of constant Jordan type. to appear in J. für die Reine und Ang. Math.

[5] J. Carlson, Z. Lin, D. Nakano, B. Parshall, The restricted Nullcone, Combinatorial and geometric representation theory (Seoul 2001), 51-75, Contemp. Math. 325, AMS Providence 2003.

[6] L. Chouinard, Projectivity and relative projectivity over group rings, J. Pure Appl. Algebra 7 (1976), 287-303. 
[7] D. Collingwood, M. McGovern, Nilpotent Orbits in Semisimple Lie Algebras, Van Nostrand Reinhold (1993).

[8] E. Friedlander, Geometry of infinitesimal group schemes, Documenta Mathematica 1998, Extra Vol. II, 55-65.

[9] E. Friedlander, B. Parshall, Support varieties for restricted Lie algebras, Invent. Math. 86 (1986), 553-562.

[10] E. Friedlander, J. Pevtsova, Representation-theoretic support spaces for finite group schemes, Amer. J. of Math. 127 (2005), 379-420.

[11] E. Friedlander, J. Pevtsova, Erratum: Representation-theoretic support spaces for finite group schemes, Amer. J. of Math. 128 (2006), 1067-1068.

[12] E. Friedlander, J. Pevtsova, П-supports for modules for finite group schemes over a field. to appear in Duke Math. J.

[13] E. Friedlander, A. Suslin, Cohomology of finite group scheme over a field, Invent. Math. 127 (1997), 235-253.

[14] J.C. Jantzen, Kohomologie von p-Lie-Algebren und nilpotente Elemente, Abh. Math.Sem. Univ. Hamburg 56 (1986), 191-219.

[15] J.C. Jantzen, Representations of Algebraic groups, Academic press, (1987).

[16] Semra Öztürk Kaptanoglu, A restriction theorem for $k\left[\mathbb{Z}_{2} \times \mathbb{Z}_{4}\right]$-Modules to Spanned Cyclic Subgroups and Multiplicities Set. Preprint

[17] G. McNinch, On the centralizer of the sum of commuting nilpotent elements, J. Pure Appl. Algebra 206 (2006), no. 1-2, 123-140.

[18] D. Quillen, The spectrum of an equivariant cohomology ring: I, II, Ann. Math. 94 (1971), 549-572, 573-602.

[19] A. Suslin, E. Friedlander, C. Bendel, Infinitesimal 1-parameter subgroups and cohomology, J. Amer. Math. Soc. 10 (1997) 693-728.

[20] A. Suslin, E. Friedlander, C. Bendel Support varieties for infinitesimal group schemes, J. Amer. Math. Soc. 10 (1997), 729-759.

[21] W. Waterhouse, Introduction to affine group schemes, Graduate Texts in Mathematics, 66 Springer-Verlag, New York-Berlin, 1979.

[22] W. Wheeler, Generic module theory, J. Algebra 185 (1996), 205-228.

Department of Mathematics, Northwestern University, Evanston, IL 60208-2730

E-mail address: eric@math.northwestern.edu

Department of Mathematics, University of Washington, Seattle, WA 98195-4350

E-mail address: julia@math.washington.edu

Department of Mathematics, Northwestern University, Evanston, IL 60208-2730

E-mail address: suslin@math.northwestern.edu 